\title{
Phosphoinositide-dependent kinase 1 controls migration and malignant transformation but not cell growth and proliferation in PTEN-null lymphocytes
}

\author{
David K. Finlay, ${ }^{1}$ Linda V. Sinclair, ${ }^{1}$ Carmen Feijoo, ${ }^{1}$ Caryll M. Waugh, ${ }^{1}$ \\ Thijs J. Hagenbeek, ${ }^{2}$ Hergen Spits, ${ }^{3}$ and Doreen A. Cantrell ${ }^{1}$
}

'Division of Immunology and Cell Biology, University of Dundee, Dundee DD15EH, Scotland, UK

${ }^{2}$ Department of Molecular Biology, Genentech, Inc., South San Francisco, CA 94080

${ }^{3}$ AMC Liver Center, Academic Medical Center S1-162, 1105BK Amsterdam, Netherlands

In normal T cell progenitors, phosphoinositide-dependent kinase I (PDK1)-mediated phosphorylation and activation of protein kinase B (PKB) is essential for the phosphorylation and inactivation of Foxo family transcription factors, and also controls $T$ cell growth and proliferation. The current study has characterized the role of PDK1 in the pathology caused by deletion of the tumor suppressor phosphatase and tensin homologue deleted on chromosome 10 (PTEN). PDK1 is shown to be essential for lymphomagenesis caused by deletion of PTEN in T cell progenitors. However, PTEN deletion bypasses the normal PDK1-controlled signaling pathways that determine thymocyte growth and proliferation. PDK1 does have important functions in PTEN-null thymocytes, notably to control the PKB-Foxo signaling axis and to direct the repertoire of adhesion and chemokine receptors expressed by PTENnull $T$ cells. The results thus provide two novel insights concerning pathological signaling caused by PTEN loss in lymphocytes. First, PTEN deletion bypasses the normal PDK1controlled metabolic checkpoints that determine cell growth and proliferation. Second, PDK1 determines the cohort of chemokine and adhesion receptors expressed by PTEN-null cells, thereby controlling their migratory capacity.

\section{CORRESPONDENCE}

Doreen A. Cantrell:

d.a.cantrell@dundee.ac.uk

Abbreviations used: CMTMR, 5-(and-6)-(((4-chloromethyl)benzoyl)amino)tetramethylrhodamine; $\mathrm{DN}$, double negative; DP, double positive; KLF2, Kruppel-like factor 2; mTOR, mammalian target of rapamycin; PDK1, phosphoinositidedependent kinase 1; PI3K, phosphoinositide 3-kinase; $\mathrm{PI}(3,4,5) \mathrm{P}_{3}$, phosphoinositide $(3,4,5)$ trisphosphate; $\mathrm{PKB}$, protein kinase B; PTEN, phosphatase and tensin homologue deleted on chromosome 10; $\mathrm{SP}$, single positive.
The lipid product of phosphoinositide 3-kinases (PI3Ks), phosphatidylinositol 3,4,5-trisphosphate $\left(\mathrm{PI}[3,4,5] \mathrm{P}_{3}\right)$, controls key signaling pathways that are fundamental for the growth, proliferation, and differentiation of normal thymocytes and mature $\mathrm{T}$ lymphocytes. $\mathrm{PI}(3,4,5) \mathrm{P}_{3}$ binds to pleckstrin homology domains in multiple signaling molecules and either allosterically regulates their catalytic activity or controls their intracellular trafficking. $\mathrm{PI}(3,4,5) \mathrm{P}_{3}$ binding proteins in $\mathrm{T}$ cells include Tec family tyrosine kinases, guanine nucleotide exchange proteins for Rho family GTPases, and the essential serine/ threonine kinases phosphoinositide-dependent kinase 1 (PDK1) and protein kinase $\mathrm{B}$ (PKB) or Akt (Okkenhaug and Vanhaesebroeck, 2003). The PDK1-PKB signaling axis is fundamentally important for normal thymocytes because it regulates protein synthesis, cell growth, and cell cycle progression via control of glycolysis and nutrient uptake (Rathmell et al., 2003;
Plas and Thompson, 2005; Cornish et al., 2006; Kelly et al., 2007). $\mathrm{PI}(3,4,5) \mathrm{P}_{3}$ signaling via mammalian target of rapamycin (mTOR) and Foxo family transcription factors also controls lymphocyte trafficking by determining the repertoire of adhesion and chemokine receptors expressed by T lymphocytes (Fabre et al., 2008; Sinclair et al., 2008; Kerdiles et al., 2009).

PI3K signaling is not only important for normal lymphocyte physiology but also contributes to malignancies (Yuan and Cantley, 2008). In particular, phosphatase and tensin homologue deleted on chromosome 10 (PTEN), which is a lipid phosphatase with specificity for the $3^{\prime}$ position of $\mathrm{PI}(3,4,5) \mathrm{P}_{3}$ (Wishart and Dixon, 2002), is down-regulated by constitutive signaling by Notch1 in T cell acute lymphoblastic leukemias

2009 Finlay et al. This article is distributed under the terms of an Attribution-Noncommercial-Share Alike-No Mirror Sites license for the first six months after the publication date (see http://www.jem.org/misc/terms.shtml). After six months it is available under a Creative Commons License (Attribution-Noncommercial-Share Alike 3.0 Unported license, as described at http://creativecommons .org/licenses/by-nc-sa/3.0/). 
(Sulis et al., 2008). There is also evidence for microRNAmediated suppression of PTEN in lymphoma. The genomic region encoding the miR-17-92 microRNA cluster is often amplified in lymphomas, and miR-17-92 down-regulates expression of PTEN (Mendell, 2008; Xiao et al., 2008). PTEN is also deleted or mutated in a diverse range of solid human tumors (Cantley and Neel, 1999), and its role as a tumor suppressor has been confirmed by genetic studies in mice (Suzuki et al., 2008). PTEN haploinsufficient mice accordingly develop a wide range of tumors, including a high frequency of $\mathrm{T}$ lymphomas (Suzuki et al., 2008). Moreover, tissue-specific deletion of PTEN in hematopoietic stem cells or thymocytes using Cre-loxP strategies results in T leukemogenesis or lymphomagenesis (Suzuki et al., 2001; Hagenbeek et al., 2004; Ma et al., 2005; Yilmaz et al., 2006; Hagenbeek and Spits, 2008).

PTEN-null lymphoma/leukemic cells are large blastoid cells indicative of unrestrained activation of metabolic pathways (Suzuki et al., 2001; Hagenbeek et al., 2004; Hagenbeek and Spits, 2008). They are also very invasive and infiltrate peripheral tissues rather than restricting their homing to lymphoid organs. PTEN-null tumors accumulate $\operatorname{PI}(3,4,5) \mathrm{P}_{3}$ and strongly activate PDK1-PKB signaling pathways. Moreover, PTEN haploinsufficient mice show reduced development of spontaneous tumors in many tissues if $\mathrm{PKB}$ alleles are deleted (Chen et al., 2006) or if PDK1 expression is reduced (Bayascas et al., 2005; Chen et al., 2006). There is thus evidence that PDK1 is important for the tumorogenesis caused by PTEN deletion, but the reason why PDK1 is important has not been explored. In normal T lymphocyte physiology, PDK1 and PKB control cell growth and proliferation (Hinton et al., 2004; Fayard et al., 2007; Juntilla et al., 2007; Kelly et al., 2007; Mao et al., 2007). It is therefore assumed that constitutive PDK1-PKB signaling mediates the unrestrained metabolism, cell growth, and proliferation of PTEN-null T cells. On this basis, the PDK1-PKB signaling axis has been proposed to be a suitable target for anticancer drug therapy (Garcia-Echeverria and Sellers, 2008; Peifer and Alessi, 2009). However, PTEN deletion alone does not result in cancer (Xue et al., 2008). PTEN-null tumors thus have secondary genetic alterations that are necessary for malignancy (Guo et al., 2008; Hagenbeek and Spits, 2008; Xue et al., 2008). Accordingly, the abnormal cell growth and proliferation of PTEN-null cells may not be a direct consequence of $\mathrm{PI}(3,4,5) \mathrm{P}_{3}$ signaling, but mediated by other pathways.

Understanding signaling pathways in nontransformed PTEN-null cells is crucial to understand how malignancy develops in cells with somatic loss or mutation of PTEN. Understanding the important signaling pathways in PTEN-null cells in vivo is also informative for the design of tumor therapies. Accordingly, the aim of this study was to explore the role of PDK1 and PKB after PTEN deletion in an in vivo mouse model of $\mathrm{T}$ lymphomagenesis. The relevance of this work is that PTEN deletion causes robust activation of PDK1PKB signaling in $\mathrm{T}$ cells, but the precise role of this pathway has not been explored. It tends to be assumed that PTENnull $\mathrm{T}$ cells will just recapitulate the normal PDK1-PKB functions where PDK1 and PKB control nutrient receptor expression, cell growth, and proliferation. Our studies show that PTEN-null $\mathrm{T}$ cell progenitors cannot transform or develop into invasive and fatal $\mathrm{T}$ lymphoma without PDK1. However, PDK1 and PKB did not control the pathological growth and proliferation of PTEN-null thymocytes. There was nevertheless an important role for PDK1, after PTEN deletion, to control the phosphorylation and activity of Foxo family transcription factors. PDK1 signaling also controlled expression of chemokine and adhesion receptors in PTENnull $\mathrm{T}$ cells and dictated their migratory capacity.

\section{RESULTS \\ PTEN loss induces T lymphomagenesis via PDK1-dependent pathways}

We used a mouse model to explore the role of PDK1 and $\mathrm{PKB}$ in $\mathrm{T}$ cell lymphomagenesis caused by PTEN deletion. Mice with PTEN alleles floxed by loxP Cre excision sequences were backcrossed to Lck-cre transgenic mice that express the Cre recombinase selectively in $\mathrm{T}$ cell progenitors in the thymus (Suzuki et al., 2001; Hagenbeek et al., 2004). These mice die at 13-14 wk because of aggressive $\mathrm{T}$ cell lymphomas (Suzuki et al., 2001; Hagenbeek et al., 2004; Hagenbeek and Spits, 2008). Fig. 1 A shows that the PTEN-null T lymphoma cells that develop in Pten $^{A / / f}$ Lck-cre mice have high constitutive levels of PDK1-PKB signaling compared with wild-type thymocytes as indicated by their high levels of PKB molecules phosphorylated on serine 473 and the PDK1 substrate threonine 308 (Fig. 1 A). There are also high levels of phosphorylated PKB substrates Foxo1, 3a, and 4 in the PTEN-null T lymphomas (Fig. 1 A). In normal thymocytes, activation of $\mathrm{PKB}$ controls nutrient uptake and cell growth by inducing expression of nutrient receptors such as the transferrin receptor (CD71) and CD98, a key subunit of L-type amino acid transporters (Cornish et al., 2006; Kelly et al., 2007). The majority of wild-type thymocytes, which do not contain active $\mathrm{PKB}$, are thus predominantly small quiescent cells that lack expression of CD71 and CD98. In contrast, the T lymphoma cells isolated from Pten ${ }^{\text {l/f }}$ Lck-cre mice are uniformly large blastoid cells that express high levels of CD71 and CD98 (Fig. 1 B).

To address the importance of PDK1 for T cell lymphomagenesis caused by PTEN deletion, we analyzed tumor development in mice genetically engineered to simultaneously delete floxed PTEN and PDK1 in T cell progenitors. In these mice, the $\mathrm{p} 56^{\text {lk }}$ proximal promoter induces cre expression in $\mathrm{T}$ cell precursors before expression of the MHC receptors CD4 and CD8 and completes deletion of floxed alleles by the double negative (DN) 3/4 stages of thymocyte development. Fig. 1 C compares survival kinetics of Pten ${ }^{A / A l}$ Lck-cre mice and Pten ${ }^{\text {Alf }}$ $P D K 1^{\text {flfl }}$ Lck-cre mice. Control wild-type mice remained healthy during the course of the study and typically have a life span of $>24$ mo (unpublished data). Pten ${ }^{f / f}$ Lck-cre mice developed massive $\mathrm{T}$ lymphomas and had a mean life span of 13-14 wk (Fig. 1 C; Hagenbeek et al., 2004; Hagenbeek and Spits, 2008). When PTEN was deleted in T cell progenitors 
engineered to simultaneously delete PDK1, the majority of mice survived with no clinical signs of lymphomagenesis (Fig. $1 \mathrm{C}$ ). A low frequency of older Pten ${ }^{f / f l} P D K 1^{f / f}$ Lck-cre mice did develop $\mathrm{T}$ lymphomas, but these tumors consisted of cells that had failed to delete PDK1 alleles (Fig. 1 D). No PDK1-null tumors were ever observed in Pten ${ }^{f / f l} P D K 1^{f / f l}$ Lck-cre mice. Normally, PDK1-floxed alleles are efficiently deleted in T cell progenitors by the lck-driven Cre transgene (Fig. 1 D). However, the observation that the very rare tumors that develop in Pten $^{f / f l} P D K 1^{f / A l}$ lck-Cre mice always contain nondeleted PDK1 alleles reveals that lymphomagenesis could only occur in PTEN-null cells that escaped PDK1 deletion.

PDK1 is obligatory for growth and proliferation of wild-type, but not PTEN-null, thymocytes

In normal T cell progenitors, PDK1 has essential functions in cell growth, proliferation, and differentiation (Hinton et al.,
2004; Kelly et al., 2007). In particular, PDK1 and PKB induce and maintain expression of the nutrient receptors CD71 and CD98, which control transferrin and amino acid uptake (Kelly et al., 2007). PKB also coordinates glucose uptake in $\mathrm{T}$ cells (Rathmell et al., 2003; Ciofani and Zuniga-Pflücker, 2005; Kelly et al., 2007; Jacobs et al., 2008). In the absence of PDK1 or PKB, thymocytes cannot increase metabolism to match the energy demands of proliferation and hence they atrophy, go through cell cycle arrest, and ultimately die (Juntilla et al., 2007; Kelly et al., 2007; Mao et al., 2007). In this context, Xue and colleagues (Xue et al., 2008) have suggested that normal thymocyte development is integral to lymphomagenesis in PTEN-null thymocytes (Xue et al., 2008). The PDK1 requirement for transformation of PTEN-null thymocytes could therefore reflect that PDK1 activation of PKB is necessary for nutrient receptor expression, cell growth and proliferation and thymic development of PTEN-deleted cells.

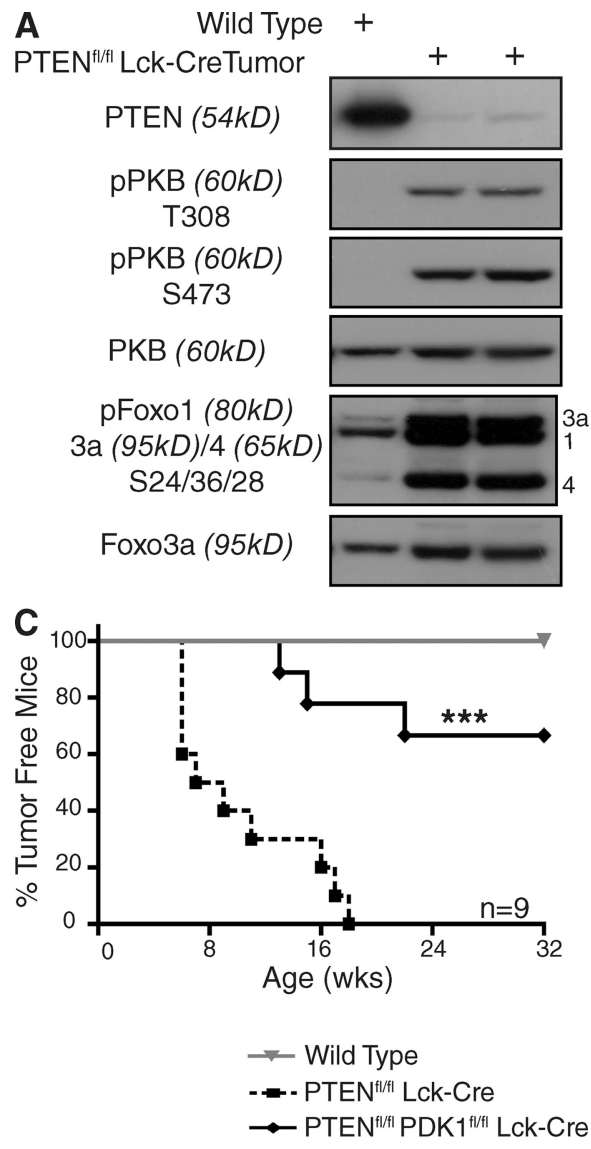

B
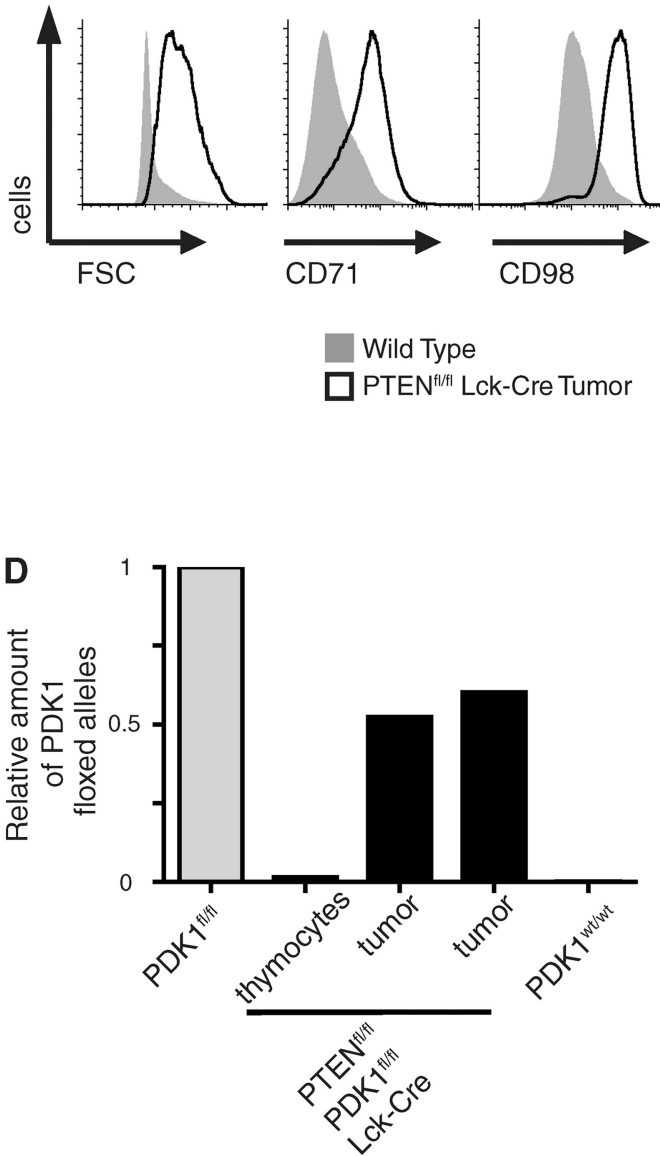

Figure 1. PTEN loss induces T lymphomagenesis via PDK1-dependent pathways. (A) Western blot analysis of PKB and Foxo 1, 3a, and 4 phosphorylation in Pten ${ }^{f l / f}$ Lck-cre thymic lymphoma cells versus total wild-type thymocytes. Data are representative of two separate experiments, three thymic lymphomas. (B) Cell size (FSC) and the expression of nutrient receptors CD71 and CD98 in total wild-type thymocytes compared with Pten $n^{f / f f}$ Lck-cre thymic lymphoma cells. Data are representative of three to five separate experiments; CD71 and CD98 (three mice), FSC (five mice). (C) Graph showing the

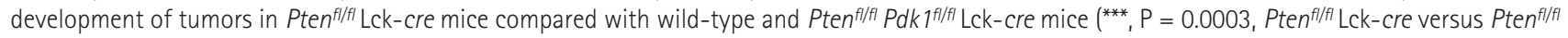

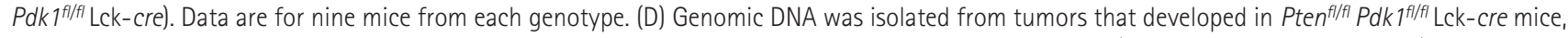
and deletion of the floxed PDK1 allele was assessed by quantative real-time PCR. Genomic DNA from Pdk $7^{f / f f}$ (minus Lck-cre) and Pdk ${ }^{W T / W T}$ mice were used as positive and negative controls, respectively. Data for tumors that developed in two separate Pten ${ }^{f / f f} P d k^{f / f f l}$ Lck-cre mice. 
To explore this possibility, we first assessed whether PTEN deletion and PKB activation was sufficient to induce growth (increase in cell mass) in $\mathrm{T}$ cells. Fig. $2 \mathrm{~A}$ shows that nontransformed thymocytes isolated from Pten ${ }^{\text {Al/f }}$ Lck-cre mice have constitutively high levels of phosphorylated PKB and phosphorylated PKB substrates Foxo1, 3A, and 4. However, PTEN-null thymocytes are only slightly larger and have only slightly higher protein content compared with wildtype control cells and are predominantly small cells compared with PTEN-null T lymphoma cells (Fig. 2, B and C). PTENnull thymocytes also fail to express universally high levels of CD71 and CD98, as seen on PTEN-null T lymphoma cells (Fig. 2 B). PDK1-mediated activation of PKB is thus not sufficient to deregulate thymocyte growth.
In further experiments, we explored whether PDK1 is required for the growth and proliferation of PTEN-null cells. As discussed, in wild-type T cells, PDK1 is essential for cell growth and proliferation. We therefore compared cell size, proliferation, and differentiation of wild-type, PDK1-null, or PTEN/PDK1-null thymocytes isolated from $P D K 1^{+/+}$

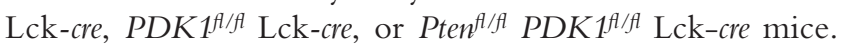
During normal thymocyte development, there are phases where cells undergo rapid proliferation and accordingly have increased metabolism and cell size. One such phase occurs in DN4 thymocytes, which are large blastoid cells that express high levels of CD71 and CD98 (Fig. 2 D; Kelly et al., 2007). In contrast, and as described previously (Kelly et al., 2007), PDK1-null DN4 thymocytes are abnormally small with low
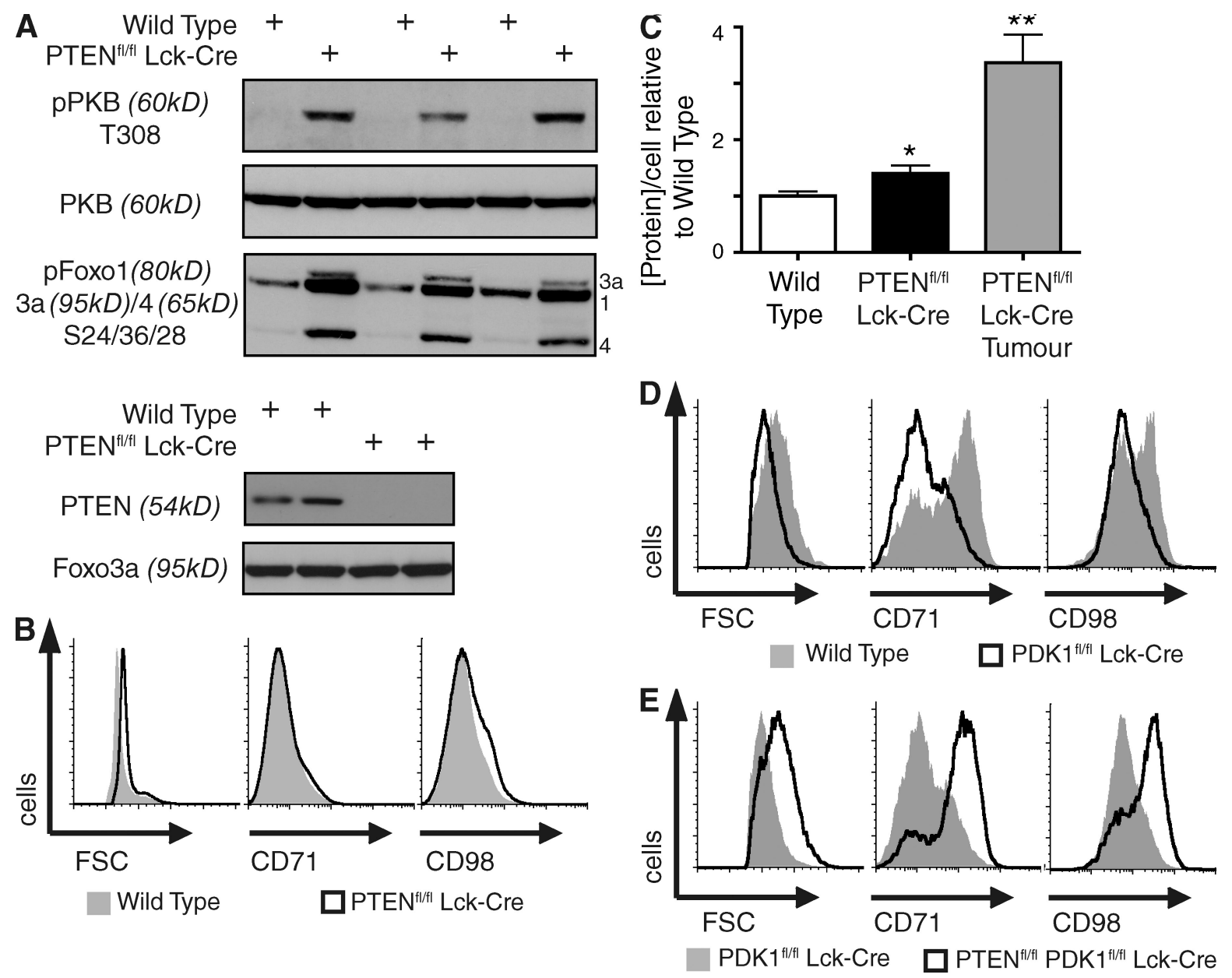

Figure 2. PDK1 is obligatory for growth and proliferation of wild-type, but not PTEN-null, thymocytes. (A) Western blot analysis of PKB and Foxo1, 3a, and 4 phosphorylation in Pten ${ }^{f / f}$ Lck-cre versus wild-type total thymocytes (top and bottom). Data are representative of four separate experiments, six sets of mice. (B) Cell size (FSC) and the expression of nutrient receptors CD71 and CD98 in total wild-type thymocytes compared with total Pten ${ }^{f / f f}$ Lck-cre thymocytes. Data are representative of three to seven separate experiments; CD71 and CD98 (three mice), FSC (seven mice). (C) Protein concentration per cell, as determined by the Bradford assay, in wild-type, Pten ${ }^{f / f f}$ Lck-cre total thymocytes, and Pten ${ }^{f / f}{ }^{\prime / L}$ Lck-cre thymic lymphoma cells $\left({ }^{*}, P<0.05 ;{ }^{* *}, P<0.01\right.$, vs. wild-type). Data are mean \pm SEM of protein extracts made from three to seven separate experiments; Pten ${ }^{f / f f}$ Lck-cre tumor (three mice), wild-type, and Pten ${ }^{f / f I}$ Lck-cre total thymocytes (seven mice). (D) Cell size (FSC) and the expression of nutrient receptors CD71 and CD98 in wild-type versus Pdk $1^{f l / f I}$ Lck-cre DN4 thymocytes. Data are representative of three separate experiments, three sets of mice. (E) Cell size (FSC) and the expression of nutrient receptors CD71 and CD98 in Pdk $7^{f / f l}$ Lck-cre versus Pten ${ }^{f / f l} P d k 1^{f / f f l} L c k$-cre DN4 thymocytes. Data are representative of three separate experiments, three sets of mice. 
levels of the nutrient receptors CD71 and CD98 (Fig. 2 D). Strikingly, Pten ${ }^{A / f} P D K 1^{f / f l}$ Lck-cre DN4 thymocytes had upregulated expression of CD98 and CD71, and the cells had returned to their normal size (Fig. 2 E). Thus, the expression of CD98 and CD71 in wild-type DN4s is PDK1 dependent, whereas the expression of these molecules in PTEN-null DN4s is PDK1 independent.

The inability of PDK1-null DN4s to maintain nutrient receptor expression prevents the cell growth and proliferation required for $\mathrm{T}$ cell differentiation (Kelly et al., 2007). Accordingly, PDK1-null $\mathrm{T}$ cell progenitors fail to develop

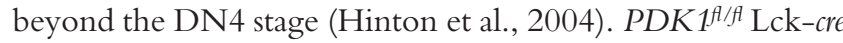
mice thus have small numbers of thymocytes compared with control mice, and these comprise mainly DNs (Fig. 3 A). Moreover, genomic PCR analysis shows that the few double positives (DPs) that appear in PDK $1^{\text {flf }}$ Lck-cre thymi have failed to delete PDK1-floxed alleles (Fig. 3 B). We therefore questioned whether the ability of PTEN deletion to induce expression of nutrient receptors and restore cell size of PDK1null DN4s restored the capacity of this subset to proliferate and to generate DP and single-positive (SP) thymocytes? Here, the data show Pten ${ }^{\text {flfl }}$ PDK $1^{\text {fl/fl }}$ Lck-cre thymocyte numbers to be in the normal range (Fig. 3 A). PTEN deletion thus induces the growth and proliferation of $\mathrm{T}$ cell progenitors independently of PDK1. Deletion of PTEN also induces $\mathrm{DN}$ to DP differentiation and the differentiation of mature SP thymocytes in the absence of PDK1 (Fig. 3 A). Moreover, peripheral $\mathrm{T}$ cell numbers in $P t e n^{f / f} P D K 1^{f / f l}$ Lck-cre mice are normal (Fig. 3 C).

The restoration of cell size, proliferation, and differentiation of Pten $^{\text {fl/f }} P D K 1^{\text {t/fll }}$ Lck-cre thymocytes could be explained if loss of PTEN changed the timing of PDK1 deletion. A series of detailed and rigorous experiments have excluded this possibility. Importantly, genomic PCR analysis in Fig. $3 \mathrm{~B}$ shows that $P t e n^{A / A} P D K 1^{A / f l}$ Lck-cre DP and SP thymocytes had successfully deleted PDK1 alleles. Hence the DPs and SPs that develop in Pten ${ }^{\text {flf }} P D K 1^{\text {flf }}$ Lck-cre mice were not cells that had "escaped" excision of PDK1 alleles. Western blot analysis also confirmed that $P t e n^{A / A l} P D K 1^{A / f}$ Lck-cre thymocytes had lost both PTEN and PDK1 protein (Fig. 3 D). In PDK $1^{\mathrm{f} / \mathrm{fl}}$ Lck-Cre mice, there is loss of functional PDK1 in DN4 thymocytes (Hinton et al., 2004). The critical experiment showing that the timing of PDK1 loss is the same in $P t e n^{f / f l} P D K 1^{f / f l}$ Lck-cre mice is shown in Fig. 3 E. These data show there was functional loss of PDK1 in Pten ${ }^{\text {f/f }} P D K 1^{\text {fl/ } / \text { A }}$ Lck-cre DN4 thymocytes as judged by the absence of phosphorylated S6 ribosomal subunits in these cells (Fig. 3 E). S6 phosphorylation is mediated by the $70-\mathrm{kD}$ ribosomal $\mathrm{S} 6$ kinase 1 (S6K1) which requires phosphorylation at its "T loop" site by PDK1. S6K1 has an additional requirement for PDK1 function in that its activation is dependent on $\mathrm{PKB}$ regulation of mTOR activity. Fig. 3 E shows that S6 phosphorylation is high in wild-type DN4s, but absent after PDK1 deletion in both PTEN wild-type and PTEN-null DN4s. Therefore, PDK1 signaling is lost in Pten $^{f / f l} P D K 1^{\text {flf }}$ Lck-cre DN4 thymocytes. Hence, in normal thymocyte development, PDK1 is absolutely required for the DN-to-DP transition, and only cells that escape deletion of PDK1 can progress. In PTENnull cells, there is no such PDK1 dependency and hence no selective advantage for cells that fail to delete PDK1. PTENnull thymocytes can thus increase cell size, proliferate, and differentiate without PDK1.

\section{The proliferation of PTEN-null T cell progenitors is dependent on RhoA}

The ability of PTEN deletion to circumvent the normal PDK1/PKB requirement for $\mathrm{T}$ cell proliferation was surprising. In this context, it is known that $\mathrm{PI}(3,4,5) \mathrm{P}_{3}$ signaling can also link to signaling pathways mediated by Rac/Rho family GTPases and these are also very important regulators of thymocyte proliferation (Cleverley et al., 1999; Liliental et al., 2000; Mullin et al., 2007). The key question then is does PTEN deletion bypass all the normal signaling pathways that control $\mathrm{T}$ cell development? The importance of RhoA for thymocytes was discovered by studies of transgenic mice that express Clostridium botulinum C3-transferase under the control of T cell-specific promoters (Cleverley et al., 1999). This toxin selectively ADP-ribosylates RhoA within its effectorbinding domain and abolishes its biological function. The expression of C3-transferase under the control of the CD2 locus control region (LCR) in T cell progenitors causes these cells to block in thymocyte differentiation at the DN3 stage such that adult CD2-C3 mice have a thymic phenotype indistinguishable from the phenotype of mice lacking key structural or sig-

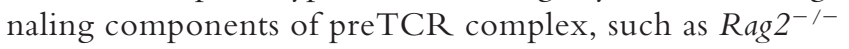
mice (Shinkai et al., 1992). We therefore assessed whether or not PTEN deletion could bypass the normal RhoA-controlled signaling pathways that control $\mathrm{T}$ cell proliferation. For these experiments, we compared the ability of PTEN deletion to induce proliferation of $\mathrm{Rag}^{2^{-/-}}$preT cells that lack expression of the preTCR complex and CD2-C3 preT cells that lack functional RhoA. The rationale for this comparison is that loss of Rag2 or inhibition of RhoA both block thymocyte differentiation at the DN3 preT cell stage. As shown previously, PTEN deletion can induce a large proliferative expansion of Rag $2^{-/-}$preT cells (Fig. 3 F; Hagenbeek et al., 2004). However, PTEN deletion could not induce proliferative expansion of CD2-C3 preT cells (Fig. 3 F). Pten ${ }^{\nexists / f l}$ Lck-cre CD2-C3 thymi are thus extremely small, comprising few cells. We conclude that PTEN deletion regulates thymocyte proliferation via RhoA-dependent pathways.

\section{PDK1 controls the phosphorylation of Foxo transcription factors in PTEN-deleted T cells}

The ability of PTEN-null thymocytes to bypass the normal $\mathrm{PDK} 1 / \mathrm{PKB}$ requirements for cell growth and proliferation raises the question of whether other PKB signaling pathways function normally after PTEN loss. Here, it is relevant that PTEN-null T lymphoma cells have high levels of phosphorylated Foxo family transcription factors Foxo1, 3a, and 4 (Fig. $1 \mathrm{~A}$ ). These transcription factors act as tumor suppressors in $\mathrm{T}$ cells, and deletion of Foxo1, 3a, and 4 mimics 

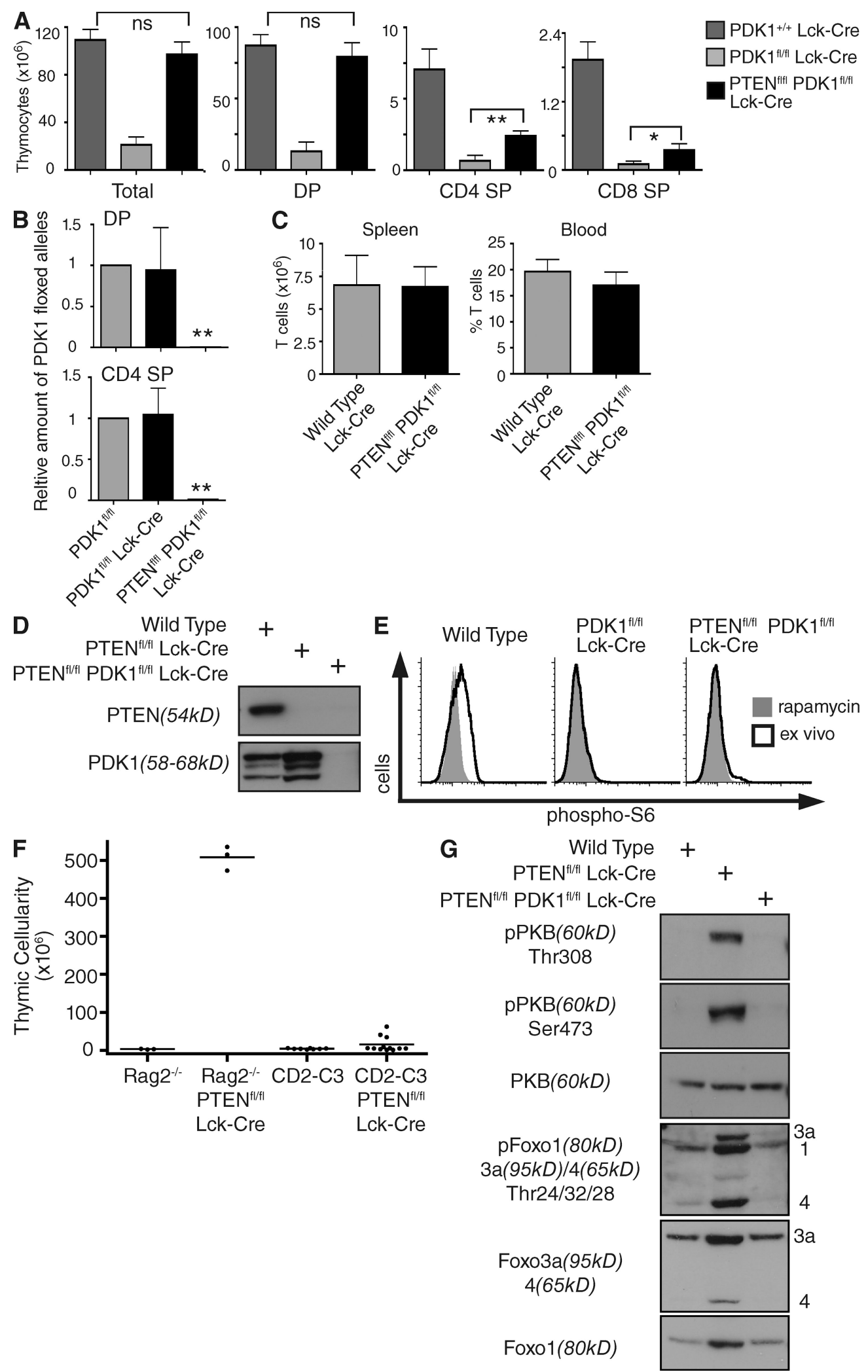

Figure 3. Thymocytes that develop in Pten ${ }^{f / f l} P d k 1^{f / f l}$ Lck-cre mice have efficiently deleted PDK1. (A) Histograms showing total and different

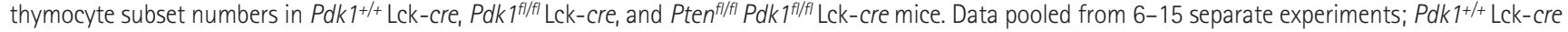
(12 mice), Pdk $7^{f / f f l}$ Lck-cre (6 mice), Pten ${ }^{f / f f} P d k 1^{f / f f}$ Lck-cre (15 mice). (B) Genomic DNA was isolated from DPs and CD4 SPs sorted from Pdk $7^{f / f f l}$ Lck-cre and Pten $^{f / f f l} P d k^{f / f f}$ Lck-cre mice, and the deletion of the floxed PDK1 allele was assessed by quantative real-time PCR. Data are from two separate experiments, 
the effect of PTEN deletion, resulting in T lymphoma development (Paik et al., 2007). The abnormal growth and proliferation of PTEN-null $\mathrm{T}$ cells is not a direct consequence of PTEN deletion. We therefore examined whether the phosphorylation of Foxos is a direct consequence of PTEN deletion and occurs in PTEN-null T cells before tumor development and whether this Foxo phosphorylation is mediated by PDK1 and PKB. Fig. 3 G compares Foxo phosphorylation in wild-type and PTEN-null thymocytes and shows that the latter contain high levels of phosphorylated Foxos. The loss of PTEN thus immediately results in Foxo phosphorylation (Fig. $3 \mathrm{G}$ ). Is the phosphorylation of Foxos in PTEN-null cells dependent on PDK1 and PKB? Fig. $3 \mathrm{G}$ shows that Pten ${ }^{A / f l} P D K 1^{\text {Alfl }}$ Lck-cre thymocytes, which have deleted both PTEN and PDK1, do not express PKB molecules phosphorylated on Thr308 or Ser473 and do not contain phosphorylated Foxo1, 3a, and 4.

\section{PTEN deletion disrupts expression of a network of adhesion molecules and chemokine receptors that control normal T cell trafficking}

The aforementioned data reveal that PTEN deletion results in PDK1/PKB-mediated Foxo1, 3a, and 4 phosphorylation in $\mathrm{T}$ cells. Is this sufficient to cause loss of Foxo transcriptional activity? In quiescent normal lymphocytes, nonphosphorylated transcriptionally active Foxos control expression of Kruppellike factor 2 (KLF2), a transcription factor that regulates expression of the chemokine receptor CCR7 and the adhesion receptor $\mathrm{CD} 62 \mathrm{~L}$ that together control $\mathrm{T}$ cell transmigration from the blood across high endothelial venules into secondary lymphoid tissues (Carlson et al., 2006; Bai et al., 2007; Fabre et al., 2008; Sebzda et al., 2008; Sinclair et al., 2008; Kerdiles et al., 2009). The expression of these receptors is also controlled by mTOR signaling (Sinclair et al., 2008), which is also triggered by PTEN loss. Accordingly, we examined PTEN-deleted T lymphoma cells and PTEN-deleted nontransformed $\mathrm{T}$ cells for expression of the Foxo-regulated targets KLF2, CD62L, and CCR7. Fig. 4 (A and B) show that normal $\mathrm{T}$ cells express KLF2, have high levels of CCR7 and CD62L mRNA expression (Fig. 4 A), and have a high concentration of CD62L and CCR7 protein expressed on their cell surface (Fig. 4 B). In contrast, PTEN-null T lymphomas do not express KLF2, CCR7, and CD62L mRNA and have dramatically reduced levels of cell surface CD62L and CCR7 (Fig. 4, A and B). It was however important to understand whether these changes were a direct consequence of PTEN deletion or a secondary consequence of cell transformation. To address this question, we examined CD62L and CCR7 expression in thymocytes isolated from Pten ${ }^{f / f l}$ Lck-cre mice before tumor development. The data show that PTEN deleted DP and SP thymocytes failed to normally up-regulate the expression of lymph node homing receptors CD62L and CCR7 (Fig. 4 C).

To determine whether the loss of these lymph node homing receptors is a direct consequence of the inactivation of Foxo transcription factors, we examined whether expression of a Foxo mutant with alanine substitutions at its PKB substrate sequences could restore expression of CD62L in PTEN-null T cells. In these experiments, we used a Foxo3a mutant with alanine substitutions at residues 32, 252, and 314 (Foxo3a AAA) that cannot be phosphorylated and inactivated by PKB (Burgering, 2008), and hence can restore Foxo transcriptional function in cells expressing active PKB. Fig. $4 \mathrm{D}$ shows that PTEN-null $\mathrm{T}$ cells have low levels of CD62L surface expression, and this remains low if cells are infected with retroviral constructs expressing either GFP alone or wild-type Foxo3a. However, cells expressing the GFP-tagged Foxo3a phospho mutant regained expression of CD62L. Therefore, restoring Foxo transcriptional activity is sufficient for surface expression of the homing receptor CD62L, indicating that inactivation of Foxo transcription factors in PTEN-null thymocytes accounts for decreased surface expression of lymph node homing receptors.

It has been proposed that KLF2 suppresses the expression of inflammatory chemokine receptors such as CCR3 and CCR 5 (Sebzda et al., 2008). Accordingly, KLF2-null quiescent peripheral $\mathrm{T}$ cells express abnormally high levels of CCR3 and CCR 5 and other inflammatory chemokine receptors. However, there was no evidence that the loss of KLF2 in PTEN-null T lymphomas or in PTEN-null nontransformed $\mathrm{T}$ cells caused abnormal expression of CCR 3 or CCR 5 (unpublished data). Nevertheless, PTEN-null T lymphomas express high levels of CD44 (Fig. 4 E; Hagenbeek and Spits, 2008), an adhesion molecule normally up-regulated by lymphocyte activation, and one that has well characterized roles controlling extravasation of normal lymphocytes during inflammatory immune responses and in controlling the homing of leukemic stem cells (DeGrendele et al., 1997; Jin et al., 2006; Krause et al., 2006; Hagenbeek and Spits, 2008). Strikingly, increased expression of CD44 was an immediate consequence of PTEN deletion. Fig. $4 \mathrm{~F}$ shows that PTEN

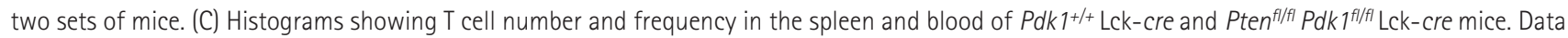
pooled from three to nine separate experiments; spleen (three sets of mice), blood (nine sets of mice). (D) Western blot analysis of PTEN and PDK1 protein

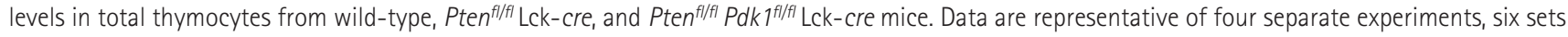

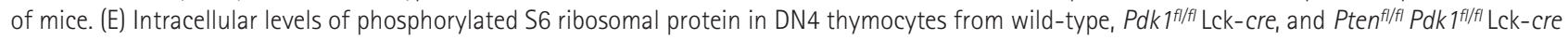
mice. DN4 thymocytes were treated with rapamycin $(20 \mathrm{nM})$ for $20 \mathrm{~min}$ to give a negative control for phospho-S6 staining. Data are representative of

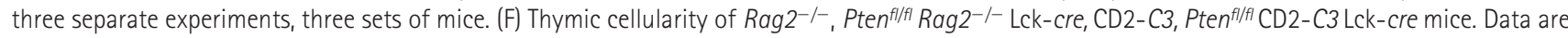

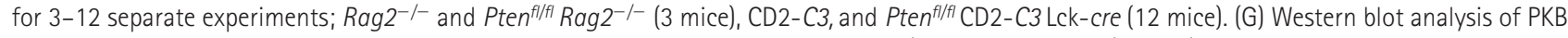
and Foxo transcription factor phosphorylation in total thymocytes from wild-type, Pten ${ }^{f / f l}$ Lck-cre, and Pten ${ }^{f / f l} P d k 1^{f / f f l}$ Lck-cre mice. Data are representative of four separate experiments, six sets of mice. 
deletion causes a universal increase in CD44 expression in thymocytes from Pten ${ }^{f / f}$ Lck-cre mice. Moreover, high levels of CD44 were sustained as PTEN-null thymocytes progressed through development and matured into peripheral $\mathrm{T}$ cells.

PDK1 regulates expression of chemokine and adhesion receptors and regulates trafficking of PTEN-null T cells to secondary lymphoid tissue

$P t e n^{f / f l} P D K 1^{f / f l}$ Lck-cre thymocytes, that have deleted both PTEN and PDK1, do not express PKB molecules phosphorylated on Thr308 or Ser473 or phosphorylated Foxo1, 3a, and 4 (Fig. $3 \mathrm{G}$ ). The deletion of PDK1 should thus restore Foxo transcriptional activity. In this context, deletion of PDK1 restored expression of the Foxo target genes CCR7 and CD62L in PTEN-null T cells and prevented up-regulation of CD44 in response to PTEN deletion (Fig. 5 A). Hence, PDK1 controls the repertoire of chemokine and adhesion receptors expressed by PTEN-deleted thymocytes; PTEN deletion can only subvert expression of CD62L, CCR7, and CD44 when PDK1 signaling is intact.

The tissue destination of lymphocytes is determined by the chemokine and adhesion receptors they express (Mora and von Andrian, 2006). CCR7 and CD62L are required for $\mathrm{T}$ cell homing to secondary lymphoid tissue, and CD44 controls lymphocyte extravasation from the blood (Arbonés et al., 1994; Förster et al., 1999; Cyster, 2005; Ebert et al., 2005;
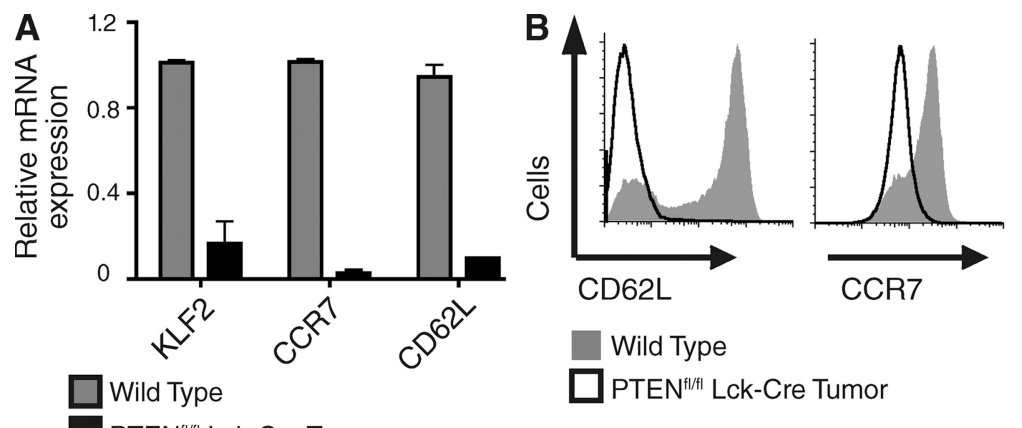

PTEN ${ }^{\text {fifl }}$ Lck-Cre Tumor
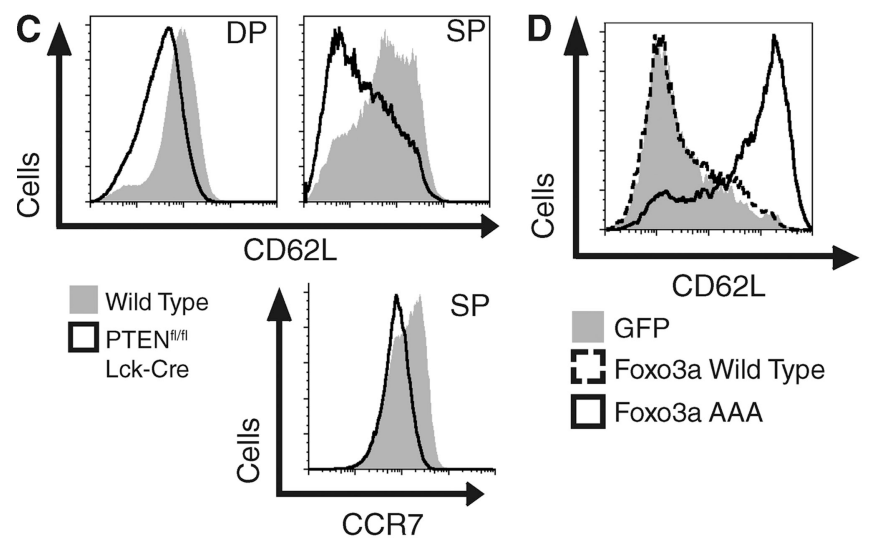

CD62L
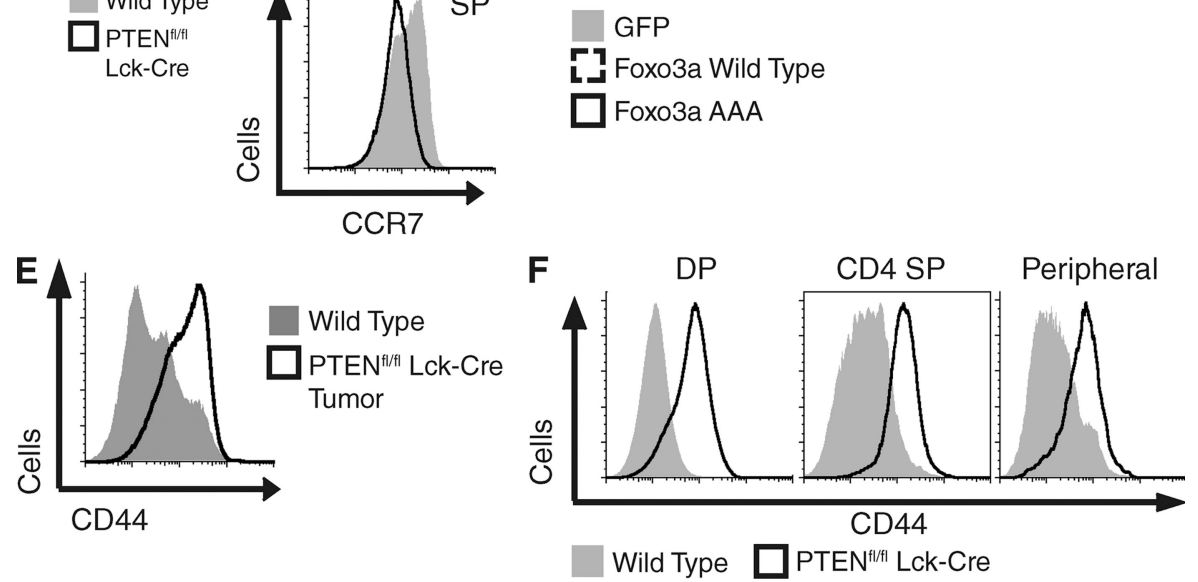

Figure 4. PTEN deletion disrupts normal expression of chemokine and adhesion receptors. (A) Analysis of mRNA expression in total wild-type thymocytes versus Pten ${ }^{f / f f}$ Lck-cre thymic lymphoma cells. (B) Expression of CD62L and CCR7 on peripheral T lymphocytes from wild-type mice versus tumor-bearing Pten ${ }^{f / / f}$ Lck-cre mice. (C) Analysis of CD62L and CCR7 expression on wild-type and Pten ${ }^{f / f}$ Lck-cre DP and CD4 SP thymocytes. (D) Pten ${ }^{f / f f l}$ Lck-cre splenic T cells were activated for $1 \mathrm{~d}$ with anti-CD3 $(0.5 \mu \mathrm{g} / \mathrm{ml})$, infected with retroviral constructs expressing GFP alone, GFP-tagged Foxo3a wildtype, or GFP-tagged Foxo3a AAA, and cultured in IL-2 $(20 \mathrm{ng} / \mathrm{ml})$ for $2 \mathrm{~d}$. GFP-positive cells were analyzed for expression of CD62L. Data are representative of two separate experiments. (E) Expression of CD44 on peripheral T lymphocytes from wild-type mice versus tumor-bearing Pten ${ }^{f l / f i} L \mathrm{Lck}$-cre mice. (F) Analysis of CD44 expression on wild-type and Pten ${ }^{f / f}$ Lck-cre DP and CD4 SP thymocytes and peripheral T lymphocytes. (A-C and E) Data are mean \pm SEM of three separate experiments, three sets of mice. 

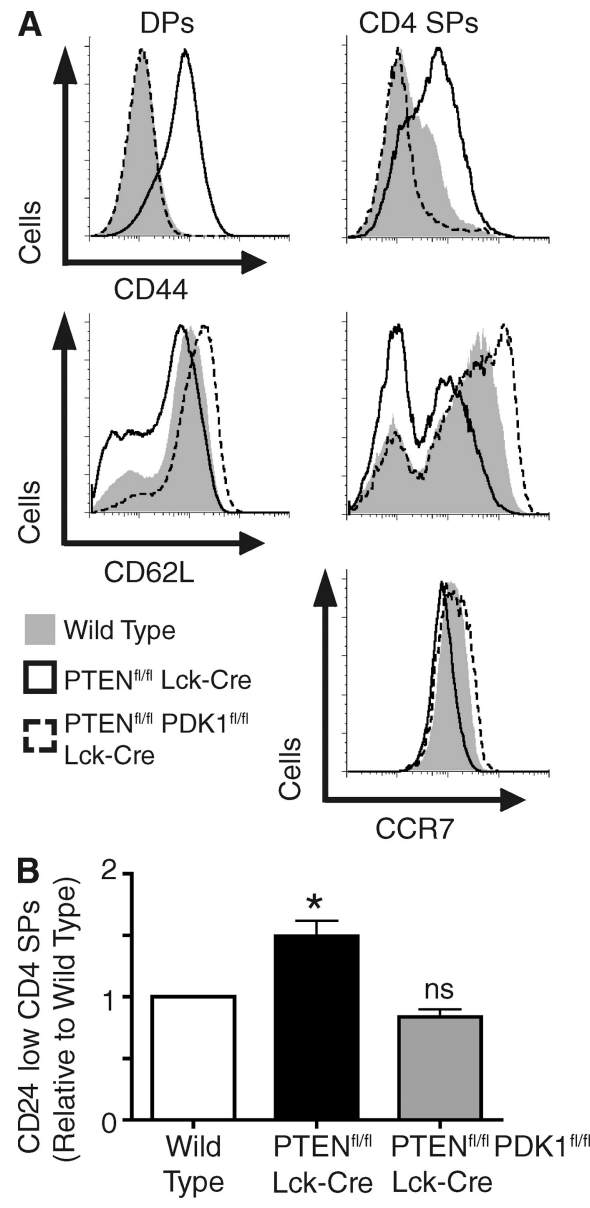

C
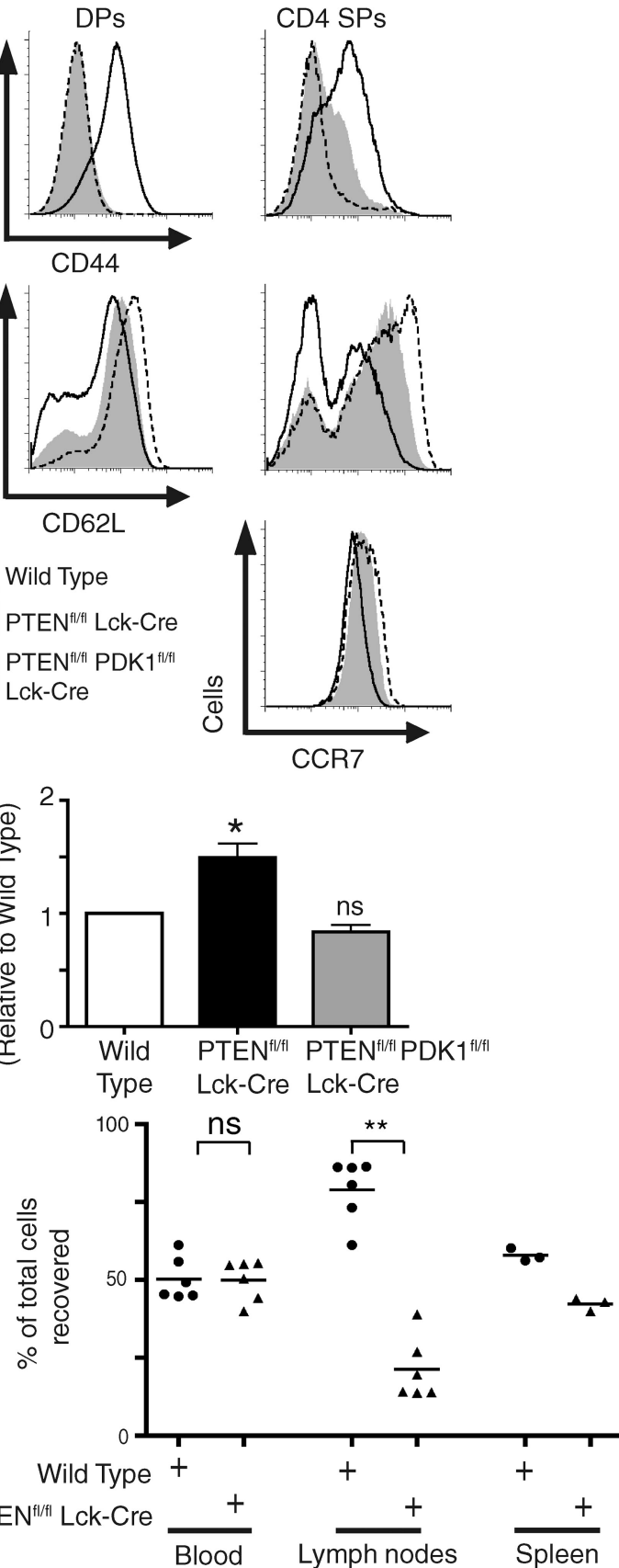

D PTEN $^{\mathrm{f} / \mathrm{l} l}$ Lck-Cre

E

PTEN $^{\mathrm{f} / / \mathrm{fl}}$ Lck-Cre

PTEN $^{f / f / f}$ PDK1 $1^{\mathrm{f} / f / 1}$ Lck-Cre
Wild Type $\overline{\text { Blood }}$

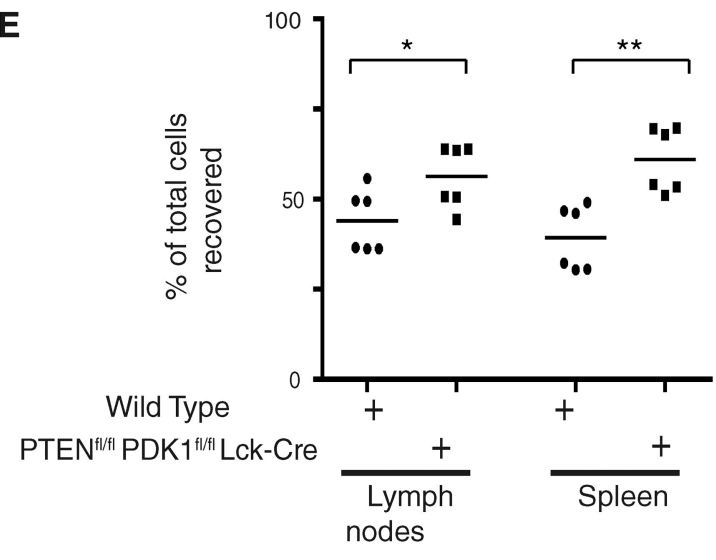

$\mathbf{F}$

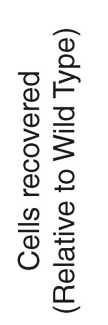

Wild Type

$\stackrel{* * *}{\Gamma^{* * *}} \stackrel{\text { ns }}{\Gamma}$

o

-

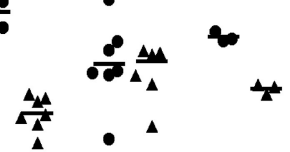

$\Delta \quad \mathbf{E} \cdot \Delta$
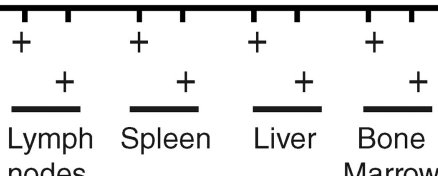

Marrow
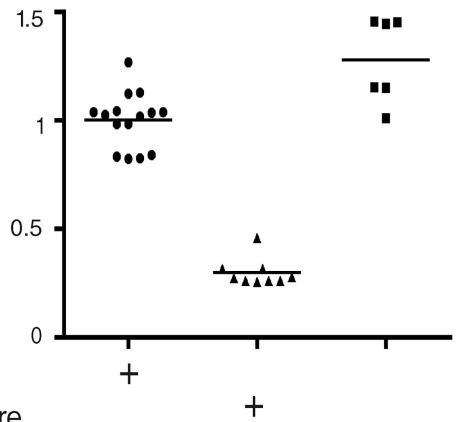

Figure 5. PDK1 and Foxo family transcription factors control the trafficking of PTEN-null T cells. (A) Analysis of the expression of CCR7,

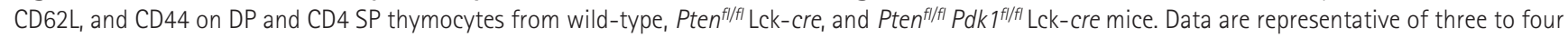
separate experiments; CCR7 (3 mice), CD62L and CD44 (4 mice). (B) Relative numbers of CD24 low mature SP thymocytes in wild-type, Pten ${ }^{f / f f}$ Lck-cre and Pten $^{f / f l} P d k 7^{f / f f}$ Lck-cre thymi ( ${ }^{*}, P<0.05$; ns, nonsignificant vs. wild-type) Data for four separate experiments, four sets of mice. (C and D) Wild-type and Pten $^{f / f f}$ Lck-cre thymocytes were stained with CMTMR and CFSE, respectively, mixed at a 1:1 ratio, and transferred in to C57BL/6 hosts. After $2 \mathrm{~h}(\mathrm{C})$ or $18 \mathrm{~h}$ (D) the mice were sacrificed and the spleen, lymph nodes, blood (C and D), liver, and bone marrow were (D) analyzed for the presence of the transferred cells. Data show the number of wild-type $(\bullet)$ or Pten ${ }^{f l / f}$ Lck-cre-labeled $(\mathbf{\Delta})$ SP thymocytes detected in each tissue (DP thymocytes in liver) as a percentage of total labeled cells recovered. Data are from three to nine mice and one to three separate experiments; $2 \mathrm{~h}$ ( 2 experiments); $18 \mathrm{~h}$; ( 3 experiments); spleen $2 \mathrm{~h}$ and bone marrow $18 \mathrm{~h}$ (1 experiment; ${ }^{* * *}, \mathrm{P}<0.001 ;{ }^{* *}, \mathrm{P}<0.01 ; \mathrm{ns}$, non significant). (E) Wild-type and Pten ${ }^{f / f l}$ Pdk $7^{f / f f}$ Lck-cre thymocytes were first analyzed to determine relative SP thymocyte numbers, then stained with CMTMR and CFSE, respectively. SP thymocytes mixed at a 1:1 ratio and transferred in to C57BL/6 hosts. After $18 \mathrm{~h}$, the mice were sacrificed and the spleen and lymph nodes were analyzed for the presence of the transferred cells. Data show the number of wild-type $(\bullet)$ or Pten ${ }^{f / f l} P d k 1^{f / f f l}$ Lck-cre-labeled $(\boldsymbol{\square})$ SP thymocytes detected in each tissue as a percentage of total labeled cells recovered. Data are from two separate experiments, six mice $\left({ }^{*}, P<0.05 ;{ }^{* *}, P<0.01\right)$. (F) Lymph node homing data from $D$ and $E$ normalized relative

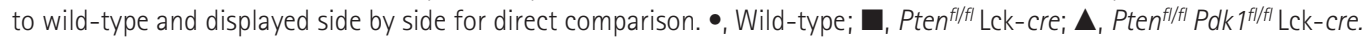


Carlson et al., 2006; Mora and von Andrian, 2006; Sebzda et al., 2008). The changes in expression of these chemokine and adhesion molecules in PTEN-null cells raises the question of whether these cells would traffic normally? In this respect, the trafficking of PTEN-null $\mathrm{T}$ cells in vivo has not been explored previously, although we have reported that PTENnull thymi contain an increased frequency of CD24 low mature SP cells (Fig. 5 B; Sinclair et al., 2008). This accumulation of mature SP cells has also been seen in thymocytes that express constitutively active PI3K (Barbee and Alberola-Ila, 2005) and reflects the observation that constitutive activation of PI3K promotes retention of mature $\mathrm{T}$ cells in the thymus. Importantly, deletion of PDK1 prevented the accumulation of CD24 low mature SP T cells in the thymus (Fig. 5 B).

What about the trafficking of PTEN-null $\mathrm{T}$ cells in the periphery? Here, it is important to note that in young PTENnull mice (4-6 wk), before tumor development, mature $\mathrm{T}$ lymphocytes are found in the spleen and lymph nodes (Xue et al., 2008). However, it is difficult to judge from this type of analysis whether PTEN-null T cells traffic normally in the periphery because $\mathrm{T}$ cell numbers in secondary lymphoid organs are determined by a balance of the following factors: the efficiency of transendothelial migration from the blood into secondary lymphoid tissue; the homeostatic proliferation of peripheral $\mathrm{T}$ cells in the lymphopenic environment found in neonates (Min et al., 2002); and the rate at which cells egress secondary lymphoid tissue (Schwab and Cyster, 2007). In this respect, the enhanced survival and proliferative properties described for PTEN-null peripheral T cells (Suzuki et al., 2001; Buckler et al., 2006) could mask any trafficking problems.

We therefore carefully examined the trafficking of PTENnull $\mathrm{T}$ cells between the blood and secondary lymphoid tissue. For these experiments, Pten ${ }^{\theta / f l}$ Lck-cre or wild-type thymocytes were labeled with either CFSE or 5-(and-6)-(((4-chloromethyl) benzoyl)amino)tetramethylrhodamine (CMTMR), mixed at a ratio of $1: 1$ and directly transferred into the blood via tail vein injections into wild-type hosts. After $2 \mathrm{~h}$, blood, lymph nodes, and spleen were analyzed for the presence of the transferred cells. This assesses the ability of the cells to undergo transendothelial migration from the blood into secondary lymphoid tissue. $2 \mathrm{~h}$ after transfer into the blood, PTEN-null $\mathrm{T}$ cells and wild-type $\mathrm{T}$ cells were equally present in the blood (Fig. $5 \mathrm{C}$ ). However, a striking difference was seen in the in vivo trafficking of PTEN-null and wild-type T cells to lymphoid tissue (Fig. 5 C). Wild-type $\mathrm{T}$ cells could enter secondary lymphoid organs such as lymph nodes and spleen, whereas the corresponding PTEN-null thymocytes were strikingly reduced in their capacity to enter lymph nodes and also had a reduced capacity to enter the spleen (Fig. $5 \mathrm{C}$ ). To further explore the trafficking of wild-type and PTEN-null cells the location of cells was explored $18 \mathrm{~h}$ after transfer into the tail veins. In these experiments, liver, blood, bone marrow, and lymphoid tissue were analyzed for the presence of the transferred cells. As with the 2-h time point, wild-type $\mathrm{T}$ cells were found in secondary lymphoid organs such as lymph nodes and spleen (Fig. 5 D), whereas the corresponding PTEN-null cells were strikingly reduced in numbers at these sites. Histological analysis showed that the few PTEN-null cells that could enter these secondary lymphoid tissues were found in the $\mathrm{T}$ cell areas (unpublished data). The PTEN-null cells had also disappeared from peripheral blood (Fig. 5 D). However, PTEN-null T cells and wildtype $\mathrm{T}$ cells were both equally present in the liver (Fig. $5 \mathrm{D}$ ) and both could home to the bone marrow (Fig. 5 D).

The accumulation of mature SP cells in PTEN-null thymi (Fig. $5 \mathrm{~B}$ ) and inability of PTEN-null $\mathrm{T}$ cells to transit from the blood to secondary lymphoid organs (Fig. 5, C and D) argues that PTEN loss can perturb the normal trafficking of $\mathrm{T}$ cells before cell transformation. This is consistent with the ability of PTEN loss to down-regulate expression of KLF2, CD62L, and CCR7. In PTEN/PDK1-null T cells expression of KLF2, CCR7, and CD62L is restored. Accordingly, we addressed whether the defects in PTEN-null T cells in terms of their ability to traffic from the blood to secondary lymphoid tissue is restored by PDK1 loss? Pten $n^{\text {flf }} P D K 1^{\text {flf }}$ Lck-cre or wild-type thymocytes were labeled with either CFSE or CMTMR, SP thymocytes mixed at a ratio of 1:1 and transferred into the tail vein of wild-type mice. After $18 \mathrm{~h}$, lymphoid tissue was analyzed for the presence of the transferred cells. Both the wild-type and PTEN/PDK1-null T cells homed effectively to secondary lymphoid organs such as lymph nodes and spleen (Fig. 5 E). Cells were also detectable recirculating in the blood (unpublished data). Indeed, PTEN/PDK1-null cells showed moderately enhanced lymph node and spleen homing capacity relative to wild-type cells. The deletion of PDK1 in PTEN-null T cells thus restores their ability to traffic to secondary lymphoid tissue (Fig. 5 F) and maintains their recirculation through the blood.

\section{DISCUSSION}

The present study shows that PDK1 is essential for lymphomagenesis caused by deletion of PTEN in T cell progenitors. In normal thymocytes, PDK1 activation of PKB is vital for cell growth and proliferation (Hinton et al., 2004; Kelly et al., 2007) because PDK1 substrates such as PKB control the essential metabolic responses that allow $\mathrm{T}$ cells to meet the energy demands of rapid proliferation. PTEN-null T lymphoma cells have high constitutive levels of PDK1/PKB signaling and are large, metabolically active blastoid cells that constitutively express high levels of transferrin receptors (CD71) and L-type amino acid transporters (CD98). In normal lymphocytes, the expression of these nutrient receptors is controlled by PDK1 and PKB. However, although PTEN deletion caused an immediate strong activation of PKB in T cell progenitors, the loss of PDK1 did not prevent expression of CD71 and CD98 in these cells. Moreover, growth and proliferation of PTEN-null $\mathrm{T}$ cell progenitors is not dependent on PDK1, indicating that these cells can sustain sufficient metabolism to support $\mathrm{T}$ cell proliferation. Collectively, these data indicate that the unrestrained growth and proliferation of PTEN-null $\mathrm{T}$ cells is not a direct effect of PTEN deletion and PKB stimulation but a secondary consequence of cell transformation. Moreover, in PTEN-null cells, the PDK1/PKB 
controlled metabolic checkpoint that functions during normal $\mathrm{T}$ cell physiology is bypassed. This result is surprising because it has been assumed that PDK1/PKB signaling pathways would be a good target for drugs to block metabolism and proliferative expansion of PTEN-null tumors (Garcia-Echeverria and Sellers, 2008; Peifer and Alessi, 2009). The present results showing that PTEN-null T cells can proliferate in vivo without PDK1/PKB signaling argue that this idea needs further evaluation. In this context, multiple genetic events contribute to transformation in PTEN-null hematopoietic cells (Xue et al., 2008) and one recurring event is the acquisition of chromosomal translocations that cause overexpression of $\mathrm{c}-\mathrm{myc}$ (Guo et al., 2008). It is known that c-myc target genes include those with essential functions for cell metabolism and cell growth (DeBerardinis et al., 2008). For example CD71, the transferrin receptor and major mediator of iron uptake in mammalian cells, is a direct c-myc target gene (O'Donnell et al., 2006). The autonomous growth of PTEN-null lymphoma cells could thus reflect that these cells have secondary genetic alterations that control cell metabolism, such as chromosomal translocations that increase cellular levels of c-myc.

In normal $\mathrm{T}$ cell progenitors, proliferation and differentiation are dependent on both PDK1- and RhoA-dependent signaling pathways (Cleverley et al., 1999; Hinton et al., 2004; Kelly et al., 2007; Mullin et al., 2007). The present data show that PTEN-deleted thymocytes remain RhoA dependent for proliferation, but can proliferate and differentiate without PDK1 and active PKB. This reveals divergence of pathological versus physiological signaling in PTEN-null cells and raises the question of why PDK1 is essential for T lymphomagenesis if PTEN deletion bypasses the normal PDK1 dependence of $\mathrm{T}$ lymphocyte proliferation? Here, the key insight from the present work is that PDK1 has an obligatory function in controlling the phosphorylation and transcriptional inactivation of Foxo1, 3a, and 4 in PTEN-null cells. These Foxo transcription factors have redundant, but essential roles controlling apoptosis after cellular stress (Burgering, 2008) and are powerful tumor suppressors in T cells (Paik et al., 2007; Tothova et al., 2007). Somatic deletion of Foxo1, $3 \mathrm{a}$, and 4 causes the rapid development of aggressive thymic lymphomas that infiltrate multiple peripheral tissues in a response that phenocopies loss of PTEN (Paik et al., 2007). Deficits in Foxo family transcriptional activity promote lymphomagenesis by promoting cell survival and resistance to oxidative stress. PDK1/PKB-induced phosphorylation and inactivation of Foxos would confer similar survival advantages that permit the subsequent genetic alterations that cooperate with PTEN deletion to cause T lymphomagenesis.

One other discovery from this study is that PDK1 dictates the repertoire of adhesion and chemokine receptors expressed by PTEN-null $\mathrm{T}$ cells. It was striking that PTEN loss downregulated expression of the transcription factor KLF2 and its gene targets: CD62L/L-selectin, which plays a fundamental role controlling transmigration of $\mathrm{T}$ cells across high endothelial venules (Arbonés et al., 1994), and the chemokine receptor CCR7, which controls lymphocyte entry and retention in lymphoid tissues (Cyster, 2005; Ebert et al., 2005; Carlson et al., 2006; Sebzda et al., 2008). PTEN loss also caused upregulation of CD44, an adhesion molecule that plays an important role in leukocyte extravasation from the blood during inflammatory immune responses and controls abnormal adhesion of malignant cells (DeGrendele et al., 1997; Jin et al., 2006; Krause et al., 2006). The present data demonstrate that the changes in the expression of trafficking receptors that occur in PTEN-null cells are mediated by PDK1 signaling. Consequently, deletion of PDK1 restored expression of CD62L and CCR7 and prevented the up-regulation of CD44 in PTEN-deleted T cells. Importantly, PTEN-null T cells also showed defective trafficking from blood into secondary lymphoid tissue, but this defect was rescued by removal of PDK1. PDK1 thus controls the migratory capacity of PTEN-null $\mathrm{T}$ cells. In this context, PTEN induced T lymphomas are invasive tumors that originate in the thymus but cause morbidity when they disseminate to the periphery and extensively infiltrate multiple tissues including the spleen, liver, kidney, and bone marrow (Suzuki et al., 2001; Hagenbeek et al., 2004). The previous idea of how PTEN might control cell adhesion and motility is that $\mathrm{PI}(3,4,5) \mathrm{P}_{3}$ signaling regulates the GTPases $\mathrm{Rac}$ and RhoA and coordinates actin and microtubule dynamics (Reif et al., 1996; Liliental et al., 2000). PTEN also has protein phosphatase activity that inhibits cell motility (Raftopoulou et al., 2004; Leslie et al., 2007). However, lymphocytes are intrinsically highly motile cells whose tissue destination is dictated by the subset of chemokine and adhesion receptors they express. The present results showing that PTEN deletion activates PDK1-mediated signaling pathways that dictate the cohort of chemokine and adhesion receptors expressed by $\mathrm{T}$ cells thus offers a mechanism by which PTEN loss could direct the trafficking of transformed lymphocytes. Here, it is noteworthy that the migratory behavior of PTEN T lymphoma cells is reminiscent of the trafficking behavior of immune-activated effector $T$ cells that have a reduced capacity to home to secondary lymphoid tissues and migrate preferentially to peripheral tissues. Such effector $\mathrm{T}$ cells traffic to peripheral tissues because they have high levels of $\mathrm{PI}(3,4,5) \mathrm{P}_{3} /$ PDK1/PKB signaling that inactivates Foxos and switches off expression of KLF2, CD62L, and CCR7. The unrestrained activation of a normal signaling pathway that redirects the trafficking of effector $\mathrm{T}$ cells during an immune response could therefore contribute to the changes in the migratory capacity of PTEN-null T lymphomas.

In summary, the present study has described the role of PDK1 in the pathological signaling caused by deletion of PTEN in T cell progenitors. A surprising result is that PTEN deletion results in strong activation of PDK1/PKB, but bypasses the normal PDK1 requirement for cell growth and proliferation of $\mathrm{T}$ cells. Nevertheless, an important function for PDK1 in PTEN-null $\mathrm{T}$ cells is to direct the activity of Foxo transcription factors and determines the repertoire of adhesion and chemokine receptors expressed by PTEN-null $\mathrm{T}$ cells. Hence only some physiological functions of PDK1 are recapitulated in the pathological reprogramming of cells 
that accompanies loss of PTEN. These data argue that PDK1/ PKB inhibitors may not be as useful to prevent tumor cell proliferation in vivo as thought; instead, inhibitors of RhoA signaling may be more valuable. However, the fact that PTENnull cells can be dependent on PDK1 to regulate adhesion and chemokine receptor expression could indicate that PDK1/ PKB inhibitors may be useful to prevent any abnormal tissue dissemination of PTEN-null T lymphomas. Moreover, tumor cells have to survive hypoxic condition once they disseminate to peripheral tissue and a role for PDK1/PKB in regulating cell survival under hypoxic conditions may be important. Equally, it cannot be excluded that PTEN-null tumors arise from cells that do need PDK1 for proliferation but that these are present at too low a frequency in the thymus for us to detect. However, if the aim of targeting PDK1 and PKB in tumors is to hit a metabolic checkpoint, the present results showing that PTEN can bypass a PDK1/PKB-controlled metabolic checkpoint that functions during normal $\mathrm{T}$ cell physiology could be an indication that drug strategies that target metabolic enzymes more directly would be more valuable tumor therapies. Such drugs would circumvent any issues of redundancy or compensation in terms of signaling molecules such as PDK1/PKB.

\section{MATERIALS AND METHODS}

Mice and cells. Mice were maintained in specific pathogen-free conditions under Home Office project licenses PPL60/3116 and PPL60/3812. Pten ${ }^{\text {A/f }}$ (Marino et al., 2002), PDK $1^{\text {flf }}$ (Mora et al., 2003), Lck-cre (Takahama et al., 1998), Rag2 $2^{-/-}$(Shinkai et al., 1992), and transgenic CD2-C3 (Cleverley et al., 1999) mice were bred and maintained in the Wellcome Trust Biocentre/ Transgenics Resource Unit, University of Dundee in compliance with UK Home Office Animals (Scientific Procedures) Act 1986 guidelines. Pten ${ }^{\text {A/f }}$ Lck-cre ${ }^{+/-}$and $P D K 1^{f / f l}$ Lck-cre $^{+/-}$mice were generated as described previously (Hagenbeek et al., 2004; Hinton et al., 2004). Pten $^{\text {A/f }}$ Lck-cre ${ }^{+/-}$were generated by crossing mice with floxed PTEN alleles, floxed PDK1 alleles, and mice expressing Cre recombinase under the control of the proximal p56 $6^{\text {lck }}$ promoter $\left(\mathrm{Lck}-\mathrm{Cre}^{+/-}\right)$. Pten ${ }^{\text {fl/f }} \mathrm{Lck}-\mathrm{cre}^{+/-} \mathrm{CD} 2-\mathrm{C} 3$ mice were generated by crossing $\mathrm{Pten}^{A / / A} \mathrm{Lck}_{-\mathrm{cre}}{ }^{+/-}$mice with transgenic CD2-C3 mice that selectively express the bacterial toxin $\mathrm{C} 3$-transferase in thymocyte progenitors under the control of CD2 promoter. Wild-type mice used as controls were either wild-type Lck-cre ${ }^{+-}$or mice negative for Cre recombinase with various PTEN and PDK1 floxed alleles. When studying normal PTEN-null T cells, to ensure the absence of transformed $\mathrm{T}$ cells, experiments were performed using mice between 4 and $5 \mathrm{wk}$ of age. Rag2 ${ }^{-/-}, \operatorname{Rag}^{{ }^{-/-}}$Pten $^{\text {A/fl }} \mathrm{Lck}-\mathrm{Cre}^{+/-}$, CD2-C3, and CD2-C3 Pten ${ }^{A / A}$ Lck-cre ${ }^{+/-}$mice used were 6 wk of age.

Flow cytometric analysis. Antibodies conjugated to FITC, PE, allophycocyanin (APC), and biotin were obtained from either BD or eBioscience. TriColor and APC Alexa Fluor 750-conjugated antibodies were obtained from Invitrogen. Cells were stained for surface expression of the following markers using the antibodies in parentheses: CD4 (RM4-5), CD8 (53-6.7), CD25 (7D4), CD44 (IM7), CD71 (C2), CD98 (RL388), CD62L (MEL-14), Thy1.2 (53-2.1), Thy1.1 (HIS51), TCR- $\beta$ (H57-597), CD16/CD32 (2.4G2) (Fc Block), and TCR $\gamma / \delta$ (GL3). Cells were stained with saturating concentrations of antibody in accordance with the manufacturer's instructions. For CCR7 staining, cells were labeled with mouse CCL19-Fc $\gamma$ and detected using PE conjugated anti-human Fc $\gamma$ (both from eBioscience). CD4 and CD8 DN subsets were gated by lineage exclusion of all thymocytes expressing CD4, CD8, or TCR $\gamma / \delta$. DN3s and DN4s were further defined as $\mathrm{CD} 25^{+} \mathrm{CD} 44^{-}$and $\mathrm{CD} 25^{-} \mathrm{CD} 44^{-}$thymocytes, respectively. Mature SP thymocytes were defined as Thy $-1^{+}$, TCR $-\beta^{\text {hi }}$ and positive for either CD4 or CD8 expression. Intracellular phospho-S6 levels in ex vivo thymocytes were assessed as previously described (Hinton et al., 2004).

Western blotting. Thymocytes were lysed on ice in NP-40 lysis buffer (50 mM Hepes, pH 7.4, 75 mM NaCl, 1\% Nonidet P-40, $10 \mathrm{mM}$ sodium fluoride, $10 \mathrm{mM}$ iodoacetamide, $1 \mathrm{mM}$ EDTA, $40 \mathrm{mM} \beta$-glycerophosphate, protease inhibitors, $1 \mathrm{mM}$ phenylmethylsulfonyl fluoride, and $100 \mu \mathrm{M}$ sodium orthovanadate). Protein was separated by SDS-PAGE, transferred to nitrocellulose membrane, and detected by Western blot analysis using standard techniques. Antibodies were raised in sheep against full length human Foxo1 and the $\mathrm{N}$ terminus of human Foxo3a. Total PTEN antibody was purchased from Santa Cruz Biotechnology, Inc., total PDK1 was obtained from Millipore, and all other antibodies were purchased from Cell Signaling Technologies.

Quantitative real-time PCR. RNA was purified using the RNeasy RNA purification Mini kit (QIAGEN) and reverse-transcribed using the iScript cDNA synthesis kit (Bio-Rad Laboratories). Genomic DNA was purified using the Ready PCR DNA column kit as per manufacturer's instructions (Flowgen Bioscience). Quantitative PCR was performed in 96-well plate format using iQ SYBR Green based detection (Bio-Rad Laboratories) on a BioRad iCycler. For mRNA analysis GAPDH, 18S, and HPRT mRNA levels were used for normalization and the derived values averaged. For genomic DNA analysis genomic GAPDH DNA levels were used for normalization.

Primers. PDK1 flox for: 5'-ATCCCAAGTTACTGAGTTGTGTTGGAAG-3'; PDK1 flox rev: 5'-GTATGCTATACGAAGTTATAGCTTCAGGAAG-3'; GAPDH forward: 5'-CATGGCCTTCCGTGTTCCTA-3'; GAPDH reverse: $5^{\prime}$-CCTGCTTCACCACCTTCTTGAT-3'; HPRT forward: 5'-TGATCAGTCAACGGGGGACA-3'; HPRT reverse: 5'-TTCGAGAGGTCCTTTTCACCA-3'; KLF2 forward: 5'-TGTGAGAAATGCCTTTGAGTTTACTG-3'; KLF2 reverse: 5'-CCCTTATAGAAATACAATCGGTCATAGTC-3'; CD62L forward: 5' -ACGGGCCCCAGTGTCAGTATGTG-3'; CD62L reverse: 5'-TGAGAAATGCCAGCCCCGAGAA-3'; CCR7 forward: 5'-CAGCCTTCCTGTGTGATTTCTACA-3'; CCR7 reverse: 5'-ACCACCAGCACGTTTTTCCT-3'; 18 S forward: 5'-ATCAGATACCGTCGTAGTTCCG-3'; 18S reverse: 5'-TCCGTCAATTCCTTTAAGTTTCAGC-3'.

Adoptive transfer. Thymocytes were isolated from $\mathrm{Pten}^{A / f l} \mathrm{Lck}_{\mathrm{Cr}} \mathrm{C}^{+-}$,

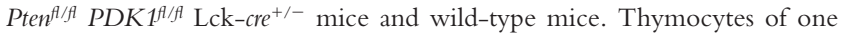
genotype were loaded with CellTracker Orange (CMTMR; Invitrogen), and the other genotype was loaded with CFSE (Invitrogen). For experiments comparing $\mathrm{Pten}^{\text {fl/f }}$ Lck-cre ${ }^{+/-}$versus wild-type, total thymocytes were mixed equally and injected into the tail vein of C57BL/6 mice. For experi-


bers of SP thymocytes were ascertained and mixed at 1:1 ratio before injection. 2 or $18 \mathrm{~h}$ after injection the mice were sacrificed and liver, blood, spleen, and lymph nodes were removed for analysis. The number of Pten ${ }^{\ell / f t}$ Lck-cre ${ }^{+/-}$or Pten $^{A / / A} P D K 1^{A / f}$ - Lck-cre ${ }^{+/-}$-labeled thymocytes recovered and the number of labeled wild-type thymocytes recovered were expressed as a percentage of the total number of recovered cells.

Virus production and $\mathrm{T}$ cell infection. Phoenix ecotropic packaging cells (Stanford University) were transfected with 5-10 $\mu \mathrm{g}$ of pBMN-GFP (Orbigen) plasmid (empty vector, Foxo3a Wild-type, or Foxo3a AAA) using a standard calcium-phosphate transfection protocol. Approximately $12-18 \mathrm{~h}$ after transfection the media was discarded and fresh media added to the dishes. After an additional $24 \mathrm{~h}$, incubation retroviral supernatants were collected and spun briefly (1,500 rpm, $5 \mathrm{~min})$ to sediment and remove packaging cells. The supernatant was transferred to fresh tubes and viral particles were concentrated by high-speed centrifugation $(20,000 \mathrm{~g}, 4 \mathrm{~h})$. After centrifugation, supernatant was discarded and concentrated viral particles were 
resuspended in $1-\mathrm{ml}$ media and stored at $-80^{\circ} \mathrm{C}$. Fresh splenocytes were activated for 12-24 h with monoclonal anti-CD3 (5 $\mu \mathrm{g} / \mathrm{ml} ; 145-2 \mathrm{C} 11$; R\&D Systems). $10^{6}$ cells $\left(2 \times 10^{6}\right.$ cells $\left./ \mathrm{ml}\right)$ were infected with freshly thawed retrovirus supernatant $(1 \mathrm{ml})$ with polybrene at $10 \mu \mathrm{g} / \mathrm{ml}$ (Sigma-Aldrich). Cells were spun at room temperature $(650 \mathrm{~g}, 45-60 \mathrm{~min})$ and cultured in RPMI-1640 medium containing L-glutamine (Invitrogen) 10\% (vol/vol) heat-inactivated FBS (Invitrogen), $50 \mu \mathrm{M} \beta$-mercaptoethanol (SigmaAldrich) and penicillin-streptomycin (Invitrogen) with $20 \mathrm{ng} / \mathrm{ml} \mathrm{IL-2}$ (Chiron) for $48 \mathrm{~h}$ before analysis of GFP positive cells.

Statistical analyses. Statistical analyses were performed using GraphPad Prism 4.00 for Macintosh, GraphPad Software. A nonparametric Mann Whitney test was used where the number of experiments performed was not sufficient to prove normal distribution. Survival curves were generated using GraphPad Prism 4.00 using the product limit method of Kaplan and Meier, which compares survival curves using the logrank test.

This work was supported by a Welcome Trust Principal Research Fellowship and Program Grant (065975/Z/01/A).

The authors have no conflicting financial interests.

\section{Submitted: 28 January 2009}

Accepted: 31 August 2009

\section{REFERENCES}

Arbonés, M.L., D.C. Ord, K. Ley, H. Ratech, C. Maynard-Curry, G. Otten, D.J. Capon, and T.F. Tedder. 1994. Lymphocyte homing and leukocyte rolling and migration are impaired in L-selectin-deficient mice. Immunity. 1:247-260. doi:10.1016/1074-7613(94)90076-0

Bai, A., H. Hu, M. Yeung, and J. Chen. 2007. Kruppel-like factor 2 controls $\mathrm{T}$ cell trafficking by activating L-selectin (CD62L) and sphingosine-1phosphate receptor 1 transcription. J. Immunol. 178:7632-7639.

Barbee, S.D., and J. Alberola-Ila. 2005. Phosphatidylinositol 3-kinase regulates thymic exit. J. Immunol. 174:1230-1238.

Bayascas, J.R., N.R. Leslie, R. Parsons, S. Fleming, and D.R. Alessi. 2005. Hypomorphic mutation of PDK1 suppresses tumorigenesis in PTEN $(+/-)$ mice. Curr. Biol. 15:1839-1846. doi:10.1016/j.cub.2005.08.066

Buckler, J.L., P.T. Walsh, P.M. Porrett, Y. Choi, and L.A. Turka. 2006. Cutting edge: $\mathrm{T}$ cell requirement for CD28 costimulation is due to negative regulation of TCR signals by PTEN. J. Immunol. 177:4262-4266.

Burgering, B.M. 2008. A brief introduction to FOXOlogy. Oncogene. 27:2258-2262. doi:10.1038/onc.2008.29

Cantley, L.C., and B.G. Neel. 1999. New insights into tumor suppression: PTEN suppresses tumor formation by restraining the phosphoinositide 3-kinase/AKT pathway. Proc. Natl. Acad. Sci. USA. 96:4240-4245. doi:10.1073/pnas.96.8.4240

Carlson, C.M., B.T. Endrizzi, J. Wu, X. Ding, M.A. Weinreich, E.R. Walsh, M.A. Wani, J.B. Lingrel, K.A. Hogquist, and S.C. Jameson. 2006. Kruppel-like factor 2 regulates thymocyte and T-cell migration. Nature. 442:299-302. doi:10.1038/nature04882

Chen, M.L., P.Z. Xu, X.D. Peng, W.S. Chen, G. Guzman, X. Yang, A. Di Cristofano, P.P. Pandolfi, and N. Hay. 2006. The deficiency of Akt1 is sufficient to suppress tumor development in Pten $+/-$ mice. Genes Dev. 20:1569-1574. doi:10.1101/gad.1395006

Ciofani, M., and J.C. Zúñiga-Pflücker. 2005. Notch promotes survival of pre- $\mathrm{T}$ cells at the beta-selection checkpoint by regulating cellular metabolism. Nat. Immunol. 6:881-888. doi:10.1038/ni1234

Cleverley, S., S. Henning, and D. Cantrell. 1999. Inhibition of Rho at different stages of thymocyte development gives different perspectives on R ho function. Curr. Biol. 9:657-660. doi:10.1016/S0960-9822(99)80289-9

Cornish, G.H., L.V. Sinclair, and D.A. Cantrell. 2006. Differential regulation of T-cell growth by IL-2 and IL-15. Blood. 108:600-608. doi:10.1182/blood-2005-12-4827

Cyster, J.G. 2005. Chemokines, sphingosine-1-phosphate, and cell migration in secondary lymphoid organs. Annu. Rev. Immunol. 23:127-159. doi:10.1146/annurev.immunol.23.021704.115628

DeBerardinis, R.J., J.J. Lum, G. Hatzivassiliou, and C.B. Thompson. 2008. The biology of cancer: metabolic reprogramming fuels cell growth and proliferation. Cell Metab. 7:11-20. doi:10.1016/j.cmet.2007.10.002
DeGrendele, H.C., P. Estess, and M.H. Siegelman. 1997. Requirement for CD44 in activated T cell extravasation into an inflammatory site. Science. 278:672-675. doi:10.1126/science.278.5338.672

Ebert, L.M., P. Schaerli, and B. Moser. 2005. Chemokine-mediated control of $\mathrm{T}$ cell traffic in lymphoid and peripheral tissues. Mol. Immunol. 42:799-809. doi:10.1016/j.molimm.2004.06.040

Fabre, S., F. Carrette, J. Chen, V. Lang, M. Semichon, C. Denoyelle, V. Lazar, N. Cagnard, A. Dubart-Kupperschmitt, M. Mangeney, et al 2008. FOXO1 regulates L-Selectin and a network of human $\mathrm{T}$ cell homing molecules downstream of phosphatidylinositol 3-kinase. J. Immunol. 181:2980-2989.

Fayard, E., J. Gill, M. Paolino, D. Hynx, G.A. Holländer, and B.A. Hemmings. 2007. Deletion of PKBalpha/Akt1 affects thymic development. PLoS One. 2:e992. doi:10.1371/journal.pone.0000992

Förster, R., A. Schubel, D. Breitfeld, E. Kremmer, I. Renner-Müller, E. Wolf, and M. Lipp. 1999. CCR7 coordinates the primary immune response by establishing functional microenvironments in secondary lymphoid organs. Cell. 99:23-33. doi:10.1016/S0092-8674(00)80059-8

Garcia-Echeverria, C., and W.R. Sellers. 2008. Drug discovery approaches targeting the PI3K/Akt pathway in cancer. Oncogene. 27:5511-5526. doi:10.1038/onc.2008.246

Guo, W., J.L. Lasky, C.J. Chang, S. Mosessian, X. Lewis, Y. Xiao, J.E. Yeh, J.Y. Chen, M.L. Iruela-Arispe, M. Varella-Garcia, and H. Wu. 2008. Multi-genetic events collaboratively contribute to Pten-null leukaemia stem-cell formation. Nature. 453:529-533. doi:10.1038/nature06933

Hagenbeek, T.J., and H. Spits. 2008. T-cell lymphomas in T-cell-specific Pten-deficient mice originate in the thymus. Leukemia. 22:608-619. doi:10.1038/sj.leu.2405056

Hagenbeek, T.J., M. Naspetti, F. Malergue, F. Garçon, J.A. Nunès, K.B. Cleutjens, J. Trapman, P. Krimpenfort, and H. Spits. 2004. The loss of PTEN allows TCR alphabeta lineage thymocytes to bypass IL-7 and Pre-TCR-mediated signaling. J. Exp. Med. 200:883-894. doi:10.1084/ jem.20040495

Hinton, H.J., D.R. Alessi, and D.A. Cantrell. 2004. The serine kinase phosphoinositide-dependent kinase 1 (PDK1) regulates T cell development. Nat. Immunol. 5:539-545. doi:10.1038/ni1062

Jacobs, S.R., C.E. Herman, N.J. Maciver, J.A. Wofford, H.L. Wieman, J.J. Hammen, and J.C. Rathmell. 2008. Glucose uptake is limiting in T cell activation and requires CD28-mediated Akt-dependent and independent pathways. J. Immunol. 180:4476-4486.

Jin, L., K.J. Hope, Q. Zhai, F. Smadja-Joffe, and J.E. Dick. 2006. Targeting of CD44 eradicates human acute myeloid leukemic stem cells. Nat. Med. 12:1167-1174. doi:10.1038/nm1483

Juntilla, M.M., J.A. Wofford, M.J. Birnbaum, J.C. Rathmell, and G.A. Koretzky. 2007. Akt1 and Akt2 are required for alphabeta thymocyte survival and differentiation. Proc. Natl. Acad. Sci. USA. 104:12105-12110. doi: $10.1073 /$ pnas. 0705285104

Kelly, A.P., D.K. Finlay, H.J. Hinton, R.G. Clarke, E. Fiorini, F. Radtke, and D.A. Cantrell. 2007. Notch-induced T cell development requires phosphoinositide-dependent kinase 1. EMBO J. 26:3441-3450. doi:10.1038/sj.emboj.7601761

Kerdiles, Y.M., D.R. Beisner, R. Tinoco, A.S. Dejean, D.H. Castrillon, R.A. Depinho, and S.M. Hedrick. 2009 Foxo1 links homing and survival of naive $\mathrm{T}$ cells by regulating $\mathrm{L}$-selectin, CCR7 and interleukin 7 receptor. Nat. Immunol. 10:176-184.

Krause, D.S., K. Lazarides, U.H. von Andrian, and R.A. Van Etten. 2006 Requirement for CD44 in homing and engraftment of BCR-ABLexpressing leukemic stem cells. Nat. Med. 12:1175-1180. doi:10.1038/ nm1489

Leslie, N.R., X. Yang, C.P. Downes, and C.J. Weijer. 2007. PtdIns(3,4,5)P(3)dependent and -independent roles for PTEN in the control of cell migration. Curr. Biol. 17:115-125. doi:10.1016/j.cub.2006.12.026

Liliental, J., S.Y. Moon, R. Lesche, R. Mamillapalli, D. Li, Y. Zheng, H. Sun, and H. Wu. 2000. Genetic deletion of the Pten tumor suppressor gene promotes cell motility by activation of Rac1 and Cdc42 GTPases. Curr. Biol. 10:401-404. doi:10.1016/S0960-9822(00)00417-6

Ma, X., A.C. Ziel-van der Made, B. Autar, H.A. van der Korput, M. Vermeij, P. van Duijn, K.B. Cleutjens, R. de Krijger, P. Krimpenfort, A. Berns, et al. 2005. Targeted biallelic inactivation of Pten in the mouse 
prostate leads to prostate cancer accompanied by increased epithelial cell proliferation but not by reduced apoptosis. Cancer Res. 65:5730-5739. doi:10.1158/0008-5472.CAN-04-4519

Mao, C., E.G. Tili, M. Dose, M.C. Haks, S.E. Bear, I. Maroulakou, K. Horie, G.A. Gaitanaris, V. Fidanza, T. Ludwig, et al. 2007. Unequal contribution of Akt isoforms in the double-negative to double-positive thymocyte transition. J. Immunol. 178:5443-5453.

Marino, S., P. Krimpenfort, C. Leung, H.A. van der Korput, J. Trapman, I. Camenisch, A. Berns, and S. Brandner. 2002. PTEN is essential for cell migration but not for fate determination and tumourigenesis in the cerebellum. Development. 129:3513-3522.

Mendell, J.T. 2008. miRiad roles for the miR-17-92 cluster in development and disease. Cell. 133:217-222. doi:10.1016/j.cell.2008.04.001

Min, B., G.D. Sempowski, and W.E. Paul. 2002. Neonates support "homeostatic" proliferation. Adv. Exp. Med. Biol. 512:91-95.

Mora, J.R., and U.H. von Andrian. 2006. T-cell homing specificity and plasticity: new concepts and future challenges. Trends Immunol. 27:235243. doi:10.1016/j.it.2006.03.007

Mora, A., A.M. Davies, L. Bertrand, I. Sharif, G.R. Budas, S. Jovanović, V. Mouton, C.R. Kahn, J.M. Lucocq, G.A. Gray, et al. 2003. Deficiency of PDK1 in cardiac muscle results in heart failure and increased sensitivity to hypoxia. EMBO J. 22:4666-4676. doi:10.1093/emboj/cdg469

Mullin, M., K. Lightfoot, R. Clarke, M. Miller, R. Lahesmaa, and D. Cantrell. 2007. The RhoA transcriptional program in pre-T cells. FEBS Lett. 581:4309-4317. doi:10.1016/j.febslet.2007.07.077

O’Donnell, K.A., D. Yu, K.I. Zeller, J.W. Kim, F. Racke, A. ThomasTikhonenko, and C.V. Dang. 2006. Activation of transferrin receptor 1 by c-Myc enhances cellular proliferation and tumorigenesis. Mol. Cell. Biol. 26:2373-2386. doi:10.1128/MCB.26.6.2373-2386.2006

Okkenhaug, K., and B. Vanhaesebroeck. 2003. PI3K-signalling in B- and T-cells: insights from gene-targeted mice. Biochem. Soc. Trans. 31:270274. doi:10.1042/BST0310270

Paik, J.H., R. Kollipara, G. Chu, H. Ji, Y. Xiao, Z. Ding, L. Miao, Z. Tothova, J.W. Horner, D.R. Carrasco, et al. 2007. FoxOs are lineagerestricted redundant tumor suppressors and regulate endothelial cell homeostasis. Cell. 128:309-323. doi:10.1016/j.cell.2006.12.029

Peifer, C., and D.R. Alessi. 2009. New anti-cancer role for PDK1 inhibitors: preventing resistance to tamoxifen. Biochem. J. 417:e5-e7. doi:10.1042/ BJ20082243

Plas, D.R., and C.B. Thompson. 2005. Akt-dependent transformation: there is more to growth than just surviving. Oncogene. 24:7435-7442. doi:10.1038/sj.onc. 1209097

Raftopoulou, M., S. Etienne-Manneville, A. Self, S. Nicholls, and A. Hall. 2004. Regulation of cell migration by the C2 domain of the tumor suppressor PTEN. Science. 303:1179-1181. doi:10.1126/science.1092089

Rathmell, J.C., R.L. Elstrom, R.M. Cinalli, and C.B. Thompson. 2003. Activated Akt promotes increased resting $\mathrm{T}$ cell size, CD28-independent $\mathrm{T}$ cell growth, and development of autoimmunity and lymphoma. Eur. J. Immunol. 33:2223-2232. doi:10.1002/eji.200324048

Reif, K., C.D. Nobes, G. Thomas, A. Hall, and D.A. Cantrell. 1996. Phosphatidylinositol 3-kinase signals activate a selective subset of Rac/ Rho-dependenteffector pathways. Curr. Biol. 6:1445-1455. doi:10.1016/ S0960-9822(96)00749-X
Schwab, S.R., and J.G. Cyster. 2007. Finding a way out: lymphocyte egress from lymphoid organs. Nat. Immunol. 8:1295-1301. doi:10.1038/ni1545

Sebzda, E., Z. Zou, J.S. Lee, T. Wang, and M.L. Kahn. 2008. Transcription factor KLF2 regulates the migration of naive $\mathrm{T}$ cells by restricting chemokine receptor expression patterns. Nat. Immunol. 9:292-300. doi:10.1038/ni1565

Shinkai, Y., G. Rathbun, K.P. Lam, E.M. Oltz, V. Stewart, M. Mendelsohn, J. Charron, M. Datta, F. Young, A.M. Stall, et al. 1992. RAG-2-deficient mice lack mature lymphocytes owing to inability to initiate $\mathrm{V}(\mathrm{D}) \mathrm{J}$ rearrangement. Cell. 68:855-867. doi:10.1016/0092-8674(92)90029-C

Sinclair, L.V., D. Finlay, C. Feijoo, G.H. Cornish, A. Gray, A. Ager, K. Okkenhaug, T.J. Hagenbeek, H. Spits, and D.A. Cantrell. 2008. Phosphatidylinositol-3-OH kinase and nutrient-sensing mTOR pathways control T lymphocyte trafficking. Nat. Immunol. 9:513-521. doi:10.1038/ ni. 1603

Sulis, M.L., O. Williams, T. Palomero, V. Tosello, S. Pallikuppam, P.J. Real, K. Barnes, L. Zuurbier, J.P. Meijerink, and A.A. Ferrando. 2008. NOTCH1 extracellular juxtamembrane expansion mutations in T-ALL. Blood. 112:733-740. doi:10.1182/blood-2007-12-130096

Suzuki, A., M.T. Yamaguchi, T. Ohteki, T. Sasaki, T. Kaisho, Y. Kimura, R. Yoshida, A. Wakeham, T. Higuchi, M. Fukumoto, et al. 2001. $\mathrm{T}$ cell-specific loss of Pten leads to defects in central and peripheral tolerance. Immunity. 14:523-534. doi:10.1016/S1074-7613(01)00134-0

Suzuki, A., T. Nakano, T.W. Mak, and T. Sasaki. 2008. Portrait of PTEN: messages from mutant mice. Cancer Sci. 99:209-213. doi:10.1111/ j.1349-7006.2007.00670.x

Takahama, Y., K. Ohishi, Y. Tokoro, T. Sugawara, Y. Yoshimura, M. Okabe, T. Kinoshita, and J. Takeda. 1998. Functional competence of $\mathrm{T}$ cells in the absence of glycosylphosphatidylinositol-anchored proteins caused by $\mathrm{T}$ cell-specific disruption of the Pig-a gene. Eur. J. Immunol. 28:2159-2166. doi:10.1002/(SICI)1521-4141(199807)28:07<2159: AID-IMMU2159>3.0.CO;2-B

Tothova, Z., R. Kollipara, B.J. Huntly, B.H. Lee, D.H. Castrillon, D.E. Cullen, E.P. McDowell, S. Lazo-Kallanian, I.R. Williams, C. Sears, et al. 2007. FoxOs are critical mediators of hematopoietic stem cell resistance to physiologic oxidative stress. Cell. 128:325-339. doi:10.1016/ j.cell.2007.01.003

Wishart, M.J., and J.E. Dixon. 2002. PTEN and myotubularin phosphatases: from 3-phosphoinositide dephosphorylation to disease. Trends Cell Biol. 12:579-585. doi:10.1016/S0962-8924(02)02412-1

Xiao, C., L. Srinivasan, D.P. Calado, H.C. Patterson, B. Zhang, J. Wang, J.M. Henderson, J.L. Kutok, and K. Rajewsky. 2008. Lymphoproliferative disease and autoimmunity in mice with increased miR-17-92 expression in lymphocytes. Nat. Immunol. 9:405-414. doi:10.1038/ni1575

Xue, L., H. Nolla, A. Suzuki, T.W. Mak, and A. Winoto. 2008. Normal development is an integral part of tumorigenesis in T cell-specific PTENdeficient mice. Proc. Natl. Acad. Sci. USA. 105:2022-2027. doi:10.1073/ pnas. 0712059105

Yilmaz, O.H., R. Valdez, B.K. Theisen, W. Guo, D.O. Ferguson, H. Wu, and S.J. Morrison. 2006. Pten dependence distinguishes haematopoietic stem cells from leukaemia-initiating cells. Nature. 441:475-482. doi: $10.1038 /$ nature 04703

Yuan, T.L., and L.C. Cantley. 2008. PI3K pathway alterations in cancer: variations on a theme. Oncogene. 27:5497-5510. doi:10.1038/onc.2008.245 